\title{
Implementation, Capabilities, and Benchmarking of Shift, a Massively Parallel Monte Carlo Radiation Transport Code ${ }^{2}$
}

Tara M. Pandya ${ }^{\mathrm{a}, 1, *}$, Seth R. Johnson ${ }^{\mathrm{a}, 1}$, Thomas M. Evans ${ }^{\mathrm{a}, 1}$, Gregory G. Davidson $^{\mathrm{a}, 1}$, Steven P. Hamilton ${ }^{\mathrm{a}, 1}$, Andrew T. Godfrey ${ }^{\mathrm{a}, 2}$

${ }^{a}$ Oak Ridge National Laboratory, 1 Bethel Valley Rd., Oak Ridge, TN 37831 U.S.A.

\begin{abstract}
This work discusses the implementation, capabilities, and validation of Shift, a massively parallel Monte Carlo radiation transport package authored at Oak Ridge National Laboratory. Shift has been developed to scale well from laptops to small computing clusters to advanced supercomputers and includes features such as support for multiple geometry and physics engines, hybrid capabilities for variance reduction methods such as the Consistent Adjoint-Driven Importance Sampling methodology, advanced parallel decompositions, and tally methods optimized for scalability on supercomputing architectures. The scaling studies presented in this paper demonstrate good weak and strong scaling behavior for the implemented algorithms. Shift has also been validated and verified against various reactor physics benchmarks, including the Consortium for Advanced Simulation of Light Water Reactors'
\end{abstract}

\footnotetext{
Notice: This manuscript has been authored by UT-Battelle, LLC, under contract DE-AC05-00OR22725 with the U.S. Department of Energy. The United States Government retains and the publisher, by accepting the article for publication, acknowledges that the United States Government retains a non-exclusive, paid-up, irrevocable, world-wide license to publish or reproduce the published form of this manuscript, or allow others to do so, for United States Government purposes.

*Corresponding Author. Tel: +1 8652413111

Email addresses: pandyatm@ornl.gov (Tara M. Pandya), johnsonsr@ornl.gov (Seth R. Johnson), evanstm@ornl.gov (Thomas M. Evans), davidsongg@ornl.gov (Gregory G. Davidson), hamiltonsp@ornl.gov (Steven P. Hamilton), godfreyat@ornl.gov (Andrew T. Godfrey)

${ }^{1}$ Radiation Transport Group, Reactor and Nuclear Systems Division

${ }^{2}$ Reactor Physics Group, Reactor and Nuclear Systems Division
} 
Virtual Environment for Reactor Analysis criticality test suite and several Westinghouse AP1000 ${ }^{\circledR}$ problems presented in this paper. These benchmark results compare well to those from other contemporary Monte Carlo codes such as MCNP5 and KENO.

Keywords: Monte Carlo methods, neutron transport, parallel computation

\section{Introduction}

The reactor physics community increasingly relies on high-fidelity transport simulations for safety analyses, reactor system upgrades, maintenance studies, and other applications. This need drives the demand for shorter solution times, increased accuracy, and more flexible capabilities in the radiation transport codes that power these simulations. Shift is a new Monte Carlo (MC) radiation transport code that has been developed in response to the growing need for flexible, fast, and accurate nuclear engineering analyses.

This work presents the development, implementation, verification, and validation of Shift. This MC code is developed and maintained at Oak Ridge National Laboratory (ORNL) by the Radiation Transport Group. Shift is the MC package in Exnihilo, a radiation transport code suite that also contains the Denovo deterministic transport code [1, 2]. Development of Shift began at ORNL in 2010 to provide a massively parallel MC code for enabling reactor analysis on the Oak Ridge Leadership Computing Facility (OLCF) high-performance computing (HPC) architectures.

Shift is designed and optimized for performing MC radiation transport calculations on current and near-future computing architectures. Shift can also run most radiation transport problems on laptops or small clusters with correspondingly reduced performance. Challenges for developing and running Shift on massively parallel computing architectures include memory management (as with all MC radiation transport codes), scaling of parallel algorithms, and tally optimization.

The implementation and benchmarking of Shift is discussed in the remainder of this paper. We describe Shift's capabilities in $\$ 2$. In $\$ 3$ we discuss validation and verification results, and in $\$ 4$ we summarize the main features of Shift and review its benchmarking results. Several features in Shift make it a unique tool for reactor analysis calculations. These include the parallel domain replication and domain decomposition algorithms discussed in \$2.8 and the tally algorithm and its scaling capabilities discussed in $\$ 2.5$ and $\$ 3.4$. 


\section{Background and Features of Shift}

Shift was designed to provide a massively parallel MC tool for reactor applications. After its initial implementation, Shift became the production MC application in the Consortium for the Advanced Simulation of Light Water Reactors (CASL) energy simulation hub [3]. Although initially developed for pressurized water reactor (PWR) analysis, Shift has been used to model other systems, including ORNL's High Flux Isotope Reactor (HFIR) [4], a very small high-flux reactor.

Shift is a general purpose radiation transport code that performs stochastic modeling of neutral particle physics using the $\mathrm{MC}$ method [5, 6]. It can perform eigenvalue calculations as well as fixed source calculations in neutron, photon, or coupled neutron-photon mode. The focus of this paper is on eigenvalue problems for reactor analysis for which Shift uses the well-known $k$-eigenvalue algorithm [6, 7, 8] described in \$2.1. The main modules of Shift include physics, tallies, geometry, source definitions, parallel decomposition, and variance reduction. These features and capabilities are discussed in the following sections.

Shift serves as the MC package of Exnihilo, the massively parallel radiation transport code suite developed and maintained at ORNL. Through this framework, Shift is combined with the deterministic radiation transport solvers in Exnihilo to take advantage of variance reduction and acceleration techniques offered by advanced hybrid methods. These hybrid methods are also discussed in the following sections.

Recently, Exnihilo has been incorporated into the SCALE code system [7], a criticality code suite developed and maintained at ORNL. The SCALE code suite has been used worldwide for over 30 years and currently has over 5600 users in over 55 countries [7]. This incorporation enables Shift to utilize the data, depletion, and sensitivity/uncertainty analysis capabilities in SCALE. In particular, the principal continuous-energy (CE) and multigroup (MG) data used in Shift comes through SCALE.

\subsection{The Monte Carlo K-Eigenvalue Method}

Here we give a brief description of the fundamental numerical methods used by Shift to model the steady-state and quasi-static neutronics behavior in operational nuclear power reactors. This material is covered in significant detail in Refs. [5, 6, 7, 8, 9]. Accordingly, only a brief discussion is given here. 
The steady-state behavior of neutrons in a nuclear reactor core is governed by the eigenvalue form of the linear Boltzmann neutron transport equation. Written in operator notation, this equation is

$$
(\hat{\mathrm{T}}-\hat{\mathrm{S}}) \psi=\frac{1}{k} \hat{\chi} \hat{\mathrm{F}} \psi
$$

where the continuous operators are defined as

$$
\begin{aligned}
\hat{\mathrm{T}} & \equiv \hat{\Omega} \cdot \nabla+\sigma_{\mathrm{t}}, \\
\hat{\mathrm{S}} & \equiv \iint \mathrm{d} \Omega^{\prime} \mathrm{d} E^{\prime} \sigma_{\mathrm{s}}\left(\hat{\Omega} \cdot \hat{\Omega}^{\prime}, E^{\prime} \rightarrow E\right), \\
\hat{\chi} & \equiv \frac{\chi(E)}{4 \pi}, \\
\hat{\mathrm{F}} & \equiv \iint \mathrm{d} \Omega^{\prime} \mathrm{d} E^{\prime} \nu \sigma_{\mathrm{f}}\left(E^{\prime}\right) .
\end{aligned}
$$

Here, $\sigma_{\mathrm{t}}$ is the total interaction cross section, $\sigma_{\mathrm{s}}$ is the differential scattering cross section, and $\sigma_{\mathrm{f}}$ is the fission cross section. The energy spectrum resulting from fission is given by $\hat{\chi}$, and $\nu$ is the average number of neutrons per fission. The fundamental unknown is the angular flux $\psi$, which is a function of space, angle, and energy. Rearranging terms in Eq. (1) yields

$$
k \psi=(\hat{\mathrm{T}}-\hat{\mathrm{S}})^{-1} \hat{\chi} \hat{\mathrm{F}} \psi \text {. }
$$

We define the fission source, $\mathrm{f} \equiv \hat{\mathrm{F}} \psi$, and multiplying by $\hat{\mathrm{F}}$ gives

$$
\hat{\mathrm{Af}}=k \mathrm{f},
$$

where $\hat{A}$ is

$$
\hat{\mathrm{A}} \equiv \hat{\mathrm{F}}(\hat{\mathrm{T}}-\hat{\mathrm{S}})^{-1} \hat{\chi}
$$

The dominant eigenvalue of Eq. (7) gives the criticality of the reactor. A self-sustaining nuclear reactor is designed to have $k=1$. We refer to the dominant eigenvalue for a reactor problem as $k_{\text {eff }}$.

Shift solves Eq. (7) using power iteration via MC method. This algorithm is simply power iteration performed using the Monte Carlo method,

$$
\begin{aligned}
\mathrm{f}^{l+1} & =\frac{1}{k^{l}} \hat{\mathrm{A}} \mathrm{f}^{l}, \\
k^{l+1} & =\frac{\int \hat{\mathrm{A}} \mathrm{f}^{l} d \Gamma}{\int \mathrm{f}^{l} d \Gamma}=k^{l} \frac{\int \mathrm{f}^{l+1} d \Gamma}{\int \mathrm{f}^{l} d \Gamma} .
\end{aligned}
$$


The fission source for the next generation is created from the previous generation. Convergence of this iteration scheme is determined by the dominance ratio, $k_{2} / k_{1}$, where $k_{2}$ is the second largest eigenvalue that satisfies Eq. (7). Thus, a standard MC power iteration solution procedure works as follows:

1. Sample an initial fission source, $f^{0}$, for cycle $l=0$

2. For $l=1, \ldots, L_{C}$,

(a) Perform $N$ random walks of fission neutrons,

(b) Sample the fission source for the next iteration, $f^{l+1}$

(c) Tally $k^{l+1}$

We need a converged fission source to ensure a true representation of the problem. Therefore, $L_{I}$ inactive cycles are executed, and these estimates of $k$ are not included in the final solution. The total number of cycles is thus split into two iteration sequences such that $L_{C}=L_{I}+L_{A}$, where $L_{A}$ is the number of active cycles. This helps reduce errors and biases in $k, \psi$, or any other physical parameter that is tallied [7, 8, 9].

\subsection{Exnihilo Front Ends}

Shift can be run through one of Exnihilo's front ends: Insilico or Omnibus. The Insilico framework is specifically designed for PWR applications, and Omnibus serves as the general HPC and general user front end.

\subsubsection{Insilico front end}

Shift is integrated into CASL's production software, the Virtual Environment for Reactor Applications (VERA) [10] via the Insilico front end. VERA executes its physics modules through a common reactor specification that defines geometry, materials, and operating conditions. This input is processed into a hierarchical extended markup language (XML) file. The Insilico package provides an executable that processes this file and executes either deterministic solvers from Denovo or the MC solver from Shift. The reactor geometry is automatically converted into Reactor Toolkit (RTK) geometry, Exnihilo's built-in reactor geometry (discussed in \$2.3). Material compositions are generated from the common material specification and $\mathrm{CE}$ data tables provided by SCALE.

Tallies are automatically generated in Insilico for pin-by-pin integrated power on user-defined axial levels. Shannon entropy tallies are also enabled by default to diagnose fission source convergence. Both of these tallies are 
performed on external, overlaid meshes that can be output for visualization purposes.

Since Insilico is only used for PWR eigenvalue problems, it produces an HDF5 file that includes $k$-code cycle information, Shannon entropy tallies, and pin powers. Integrated pin powers are produced on demand using Python postprocessing scripts in Insilico. The Insilico driver can also output the material and RTK geometry definitions for diagnostic purposes.

\subsubsection{Omnibus front end}

The Omnibus front end provides a general interface to Shift and Denovo. The Python preprocessor for Omnibus converts an ASCII input file to an $\mathrm{XML}$ input, which is then read by the $\mathrm{C}++$ driver on a single master node and distributed to all other parallel nodes. Preprocessing features include validating input parameters, setting default parameters, and running MCNP5 to preprocess an MCNP5 input file if using Shift's MCNP5 geometry support [8].

After problem setup, Denovo and Shift managers in Omnibus handle the transport solve procedure. During a Shift run, Omnibus writes mesh and cell tallies to HDF5 files if requested. If a depletion calculation is being performed, the depletion data for each step, including isotopics, burnup, collapsed cross sections, and depletable region fluxes/powers, are also written to an HDF5 file during the solution procedure.

At the end of the solving routine, Omnibus produces an XML file with diagnostic information (including a unique problem run identifier, timing information, material composition tables, and small tallies such as $k_{\text {eff }}$ estimators) as well as HDF5 and Silo data files, depending on what tallies are enabled. Built-in postprocessing (again using Python) produces plots of the $k_{\text {eff }}$ estimate and Shannon entropy per cycle, summarizes tally results, converts a subset of the HDF5 results to Excel-importable comma-separated value (CSV) files, and generates an HTML summary of the problem input and output.

\subsection{Geometry Packages}

Geometry tracking during a Shift transport calculation is performed using a geometry-agnostic interface. This enables a variety of geometry implementations to be incorporated into the Shift framework, allowing users

of common codes such as MCNP5 and KENO to reuse their existing inputs when running Shift. 
Each geometry type in Shift provides an opaque handle to the underlying state. This state handle is fully encapsulated by a geometry class that applies tracking operations (such as distance-to-boundary and changes in direction) using a common interface to the state. Currently, Shift implements the following geometries:

- SCALE geometry using the Atlas package (KENO-VI and KENO-V geometry input) [7];

- MCNP5 geometry supported through the Lava library (developed at ORNL) [11]; and

- Reactor Toolkit (RTK) geometry for PWRs native in Exnihilo.

Input files defining geometry for SCALE, MCNP5, and RTK can be included in the front end input for a Shift transport calculation. These geometry input files are parsed through different code packages. The same parser can also read material compositions associated with the cells in the geometry. The composition block in SCALE input and the material (m) cards in an MCNP5 data block are both supported through this feature. In the case of MCNP5 input, mt cards, (which specify $S(\alpha, \beta)$ treatments for materials), are also correctly captured.

The built-in RTK geometry package is tailored toward light water reactor (LWR) applications. It provides a small set of simple geometric constructs that allows rapid tracking through the multicylinder pin-cell geometries that are common in LWRs. This package was designed for CASL problems, and the geometry parsing is fully integrated into the Insilico front end. Because of the relatively simple geometric shapes this RTK package supports, it can track particles very quickly and therefore serves as a very quick and efficient tool for PWR applications. However, because of the very simple geometrical shapes it supports, the scope of problems that can be modeled using the RTK geometry package is limited.

\subsubsection{Abstract geometry interface}

Shift implements a set of accessors and interface methods for particle tracking in its application program interface (API) for geometry. As stated previously, these methods call their respective routines based upon the templated geometry type. The set of accessors implemented via the geometry API are the following: 
- cell - Return the current cell ID in which a particle resides;

- matid - Return the current material ID in which a particle resides;

- normal - Return the outward unit normal of the current surface;

- position - Return the current particle position; and

- direction - Return the current particle direction.

Along with these accessors, the interface methods take the current state as an input parameter. The main particle tracking geometry interface methods in Shift are the following:

- distance_to_boundary - Calculate the distance from the current particle position to the next surface;

- move_across_surface - Move a particle across a surface in the current particle direction;

- move_within_cell - Move a particle a given distance within a cell in the current particle direction;

- change_direction - Change the particle direction;

- reflect - Change the direction at a reflecting surface; and

- initialize - Initialize the particle with the given state.

Shift tracks a particle until it is absorbed or leaves the domain/problem boundary using these methods. The specific details of each of these methods is dependent on a particular geometric implementation; therefore, the robustness of the particle tracking in Shift depends on these packages. For example, Lava provides an interface to MCNP5 tracking routines, so using Lava will produce identical results with respect to geometry tracking as MCNP5.

\subsection{Physics Capabilities and Features}

The modular design of Shift allows the coupling of diverse physics packages. Shift has the ability to use MG or CE physics when solving a radiation transport problem. Both support neutron, photon, and coupled neutronphoton transport. 


\subsubsection{Multigroup physics}

The multigroup physics algorithm in Shift is an implementatin of the SCALE MG physics algorithm that supports fully anisotropic scattering through $\mathrm{P}_{N}$ moment expansions. MG cross sections can generally be defined in one of two ways in Shift problems. The first is to automatically build cross sections from the compositions read from an input file as described in $\$ 2.3$. The second is by manually entering tables and lists of $\Sigma_{s}, \nu \Sigma_{f}$, and $\Sigma_{t}$ for each material.

These MG cross sections can be generated directly from SCALE crosssection libraries using XSProc. For XSProc processing, the default is to treat each composition as an infinite homogeneous medium. Alternatively, a full interface to the SCALE material specification is supported so that more advanced resonance self-shielding models can be applied [7, 12].

\subsubsection{Continuous-energy physics}

The SCALE Continuous-Energy (SCE) physics module in Shift is an implementation of the CE physics used in SCALE's CE KENO code, which uses ENDF [13] data processed through AMPX [14]. The SCE physics package provides not only a physics engine but also an efficient $\mathrm{C}++$ and Python interface to SCALE CE data.

Similar to CE KENO, SCE physics does not produce separate neutrons from $(\mathrm{n}, 2 \mathrm{n})$ or other neutron production reactions. Instead, the sampling routine adjusts the weight of the outgoing particle according to the number of neutrons produced. The treatment of fission site sampling is significantly different in Shift SCE as compared to CE KENO, which stores a fission site only if a fission collision occurs [7]. In Shift, as in MCNP5, the SCE physics module samples the number of fission sites produced at every collision, regardless of the type of collision.

\subsection{Tallying Capabilities and Features}

One of the unique capabilities of Shift is an object-oriented, geometryand physics-agnostic tally system. Its design allows for speed, flexibility, and extensibility.

\subsubsection{General features}

Every tally class is built using a number of shared components that provide useful, advanced functionality. Most tallies support energy binning over neutrons, photons, or both concurrently. The tally system supports tallying 
any number of the following multipliers concurrently and independently for each tally:

- particle flux;

- reaction rates, including $\sigma_{\mathrm{t}}, \sigma_{\mathrm{a}}, \nu \sigma_{\mathrm{f}}$, etc.;

- heating rates, using MG Kinetic Energy Released per Unit Mass (KERMA) data;

- fission heat production rates, using fission $Q$ values from SCALE data; and

- arbitrary energy- and particle-dependent responses input by the user.

Currently, the user-input responses support histogram bins and point data with linear-linear, linear-log, log-linear, and log-log interpolation. A simple object-oriented interface allows new responses to be created and integrated quickly into Shift.

Each tally is given a vector of evaluated multipliers (e.g., macroscopic cross sections such as $\sigma_{\mathrm{t}}$ and $\nu \sigma_{\mathrm{f}}$ in the current material) and responses (e.g., the dose factor at the current energy). The tally in turn will take the products of the multipliers with the estimator (e.g., path length) and accumulate them during a particle history in a bin. (The bin is usually a combination of a cell and an energy group.) At the end of the history, the list of bins encountered during the history is accumulated into domain-local mean and variance arrays. Energy-integrated means and variances are calculated and stored independently of the binned values.

Shift currently uses history-based statistics to determine the accuracy of tally estimators. The estimator for reaction $x$ in cell $c$, group $g$, for history $n$, is

$$
R_{x c g n}=\frac{1}{V_{c}} \sum_{i} R_{x}\left(E_{i}\right) l_{i} w_{i} \equiv \frac{1}{V_{c}} r_{x c g n},
$$

where $V_{c}$ is the volume of cell $c$, and the summation is over each track $i$ with corresponding energy $E_{i}$, path length $l_{i}$, and weight $w_{i}$. The actual value accumulated is $r_{x c g n}$, a vector of all $x$, that is passed into the accumulate function along with the tally bin (which stores $g$ and $c$ ). The Tallier class, which provides an interface between the tallies and the transport, calculates $R_{x}\left(E_{i}\right)$ for all the reactions, $x$, and passes that vector into each Tally instance. See Fig. 1 in the following section for more details. 
At the end of the history, each Tally instance accumulates the first and second moments of each $r$ :

$$
a_{x c g} \equiv \sum_{n} r_{x c g n}, \quad b_{x c g} \equiv \sum_{n} r_{x c g n}^{2} .
$$

The estimator for all histories is

$$
R_{x c g}=\frac{1}{N} \sum_{n} R_{x c g n}=\frac{1}{N} \frac{1}{V_{c}} \sum_{n} r_{x c g n}=\frac{1}{N} \frac{1}{V_{c}} a_{x c g} .
$$

The estimated variance of the sample mean, $R_{x c g}$, can then be calculated as

$$
\begin{aligned}
\operatorname{Var}\left[R_{x c g}\right] & =\frac{1}{N} \frac{1}{N-1} \sum_{n}\left(R_{x c g n}-R_{x c g}\right)^{2} \\
& =\frac{1}{N} \frac{1}{N-1} \frac{1}{V_{c}^{2}} \sum_{n}\left(r_{x c g n}-\frac{1}{N} a_{x c g}\right)^{2} \\
& =\frac{1}{N^{2}} \frac{1}{N-1} \frac{1}{V_{c}^{2}}\left[N b_{x c g}-a_{x c g}^{2}\right] .
\end{aligned}
$$

This is expressed in terms of the first moment, $a$, and the second moment, $b$. We can also express it in terms of the calculated mean:

$$
\begin{aligned}
\operatorname{Var}\left[R_{x c g}\right] & =\frac{1}{N^{2}} \frac{1}{N-1} \frac{1}{V_{c}^{2}}\left[N b_{x c g}-N^{2} V_{c}^{2}\left(R_{x c g}\right)^{2}\right] \\
& =\frac{1}{N-1}\left[\frac{1}{N V_{c}^{2}} b_{x c g}-R_{x c g}^{2}\right] .
\end{aligned}
$$

When normalizing by a user-given multiplicative factor $k$, the mean becomes

$$
R_{x c g}=\frac{1}{N} \frac{k}{V_{c}} a_{x c g},
$$

with the variance

$$
\operatorname{Var}\left[R_{x c g}\right]=\frac{1}{N^{2}} \frac{1}{N-1} \frac{1}{V_{c}^{2}}\left[N k^{2} b_{x c g}-a_{x c g}^{2}\right] .
$$

Parallel reductions and summations are typically performed on the tallies only at the end of the entire calculation. One exception is the domain-decomposed Shannon entropy tally, which is reduced and evaluated at the end of every $k_{\text {eff }}$ cycle. Note that Shift does not account for the correlation between variates when calculating variance over iterations. 


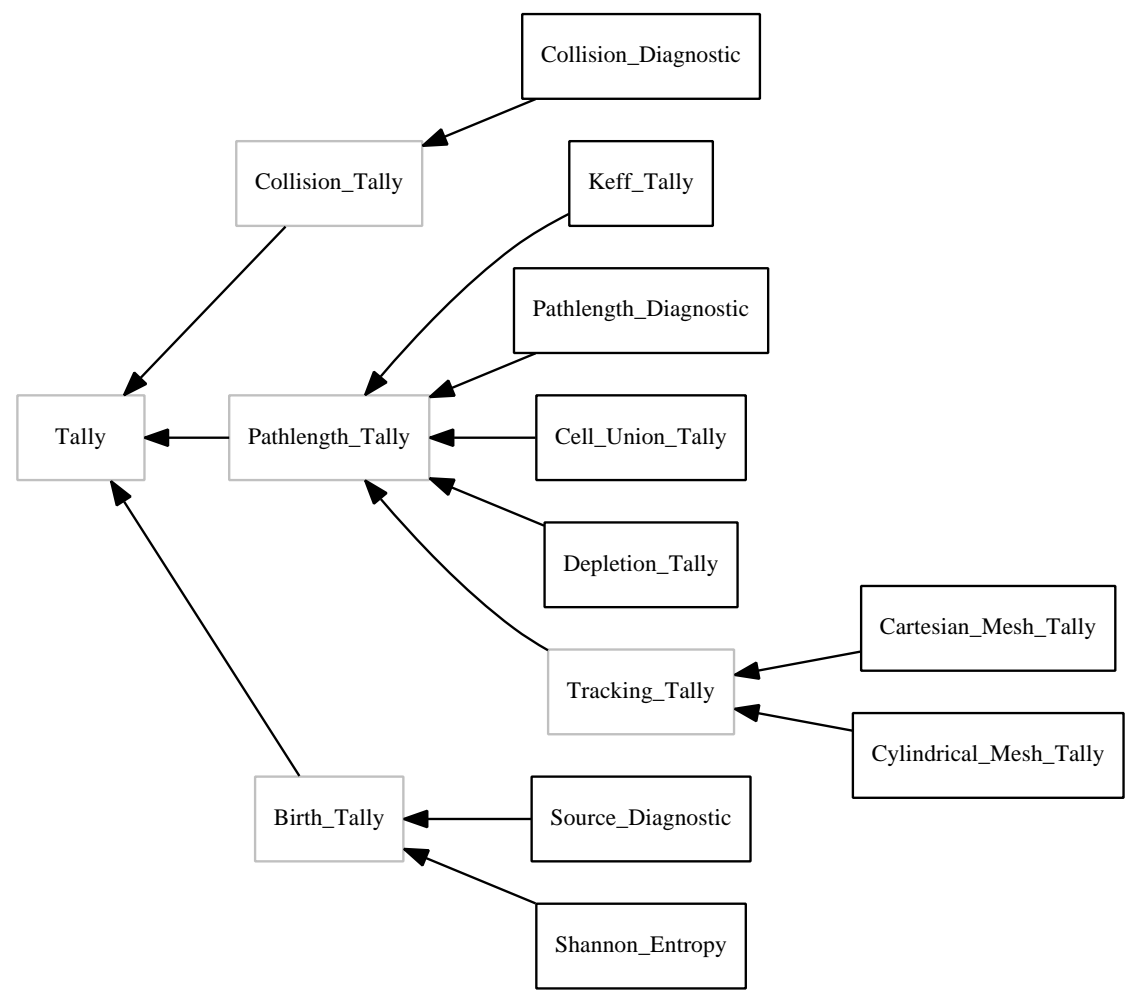

Figure 1: Existing tallies with inheritance hierarchy.

\subsubsection{Available tallies}

Shift contains a number of user-accessible tallies by default. It supports an arbitrary combination of tallies, represented in Fig. 1. The virtual classes Birth_Tally, Pathlength_Tally, Collision_Tally, and Death_Tally provide tallying interfaces for four main transport events: particles being born, streaming inside a single material, undergoing a collision, and ending a particle lifetime.

The birth tally is called with the particle's state when created, the path length tally is called with the particle's pre-streaming state and the distance it will travel, the collision tally is called with the particle's pre-collision and post-collision states, and the death tally is called with the particle's final state. The particle state comprises weight, energy, position and direction vectors, and cell and material identification numbers (IDs). The multiple tallying events plus the availability of state information enables the implementation of highly customizable tallies. 
Because the tallies use $\mathrm{C}++$ polymorphism, there is no run time penalty for having additional unused tally types. The polymorphic base class Tally enables common operations on all tallies, such as obtaining energy bins and a list of all reactions to be tallied on.

The following tallies and diagnostics (see Fig. 1) are available to the user:

Collision diagnostic. This diagnostic tallies the number of collisions per history as a function of material, nuclide, and reaction ID.

Path length estimator for $k_{\text {eff. }}$ Currently, $k_{\text {eff }}$ is calculated using a path length estimator. The path length estimate of the eigenvalue in cycle $i$ is the reaction rate for neutron production in each track length $j$ of particle history $n$ :

$$
k_{i, n, j}=\nu \sigma_{\mathrm{f}}\left(E_{j}\right) l_{j} w_{j}
$$

so that the $k_{\text {eff }}$ estimate for a single cycle is

$$
k_{i}=\frac{1}{N} \sum_{n=1}^{N} \sum_{j=1}^{J_{n}} \nu \sigma_{\mathrm{f}}\left(E_{j}\right) l_{j} w_{j} .
$$

Adding collision and absorption estimators to reduce the statistical uncertainty of the path length estimator is a work in progress.

Cell union tally. Geometry cells and unions of cells are tallied using a hash table, allowing constant-time scaling with respect to the number of total cells being tallied.

Depletion tally. The current depletion implementation in Shift folds finegroup fluxes with CE cross sections for each nuclide in each region. Because the collapsed cross sections do not have an associated statistical variance, it is not necessary to calculate flux variances for each fine-group bin, allowing memory and speed savings by omitting the variance calculation entirely.

Cartesian mesh tally. Shift supports independent mesh tallies. A Cartesian mesh can be defined over all or part of the physical problem geometry. Any path length tally can then be accumulated on the mesh. The Cartesian mesh tally is output at the end of a problem in Silo format for easy analysis using VisIt [15]. Tallying on overlaid meshes requires different communication patterns when running on spatially decomposed problems, as described in \$2.8. 
Cylindrical mesh tally. In addition to a Cartesian mesh, particles can be tracked on a translated, rotated cylinder broken into $(r, z, \theta)$ mesh cells.

Source diagnostic. A diagnostic tally is provided to calculate the source density binned into spatial cells. It also calculates the particle source density i.e., binning $n(\mathbf{r})$ rather than $w n(\mathbf{r})$ to assist in the construction of biased sources.

Shannon entropy. The Shannon entropy of the fission source distribution is a statistical metric, based on information theory, for measuring fission source convergence [16]. The Shannon entropy $H$ is calculated as

$$
H=-\sum_{m=1}^{M} f_{m} \log _{2} f_{m},
$$

where $m$ are spatial bins distributed over the problem and $f_{m}$ is the fraction of source particles born in bin $m$. A concentrated source has a low entropy (zero if all source particles are born in the same cell), and a distributed source has a high entropy $\left(\log _{2} N\right.$ if each particle is born in a different cell).

As the fission source distribution converges, the entropy will approach (and oscillate around) a problem-dependent value. Because an unconverged fission source may lead to a biased, unphysical solution, it is now common practice to use an entropy tally to determine when the source is converged and when to start tallying "active" cycles [16].

\subsubsection{Tally performance}

The tallies in Shift are designed to scale well for large problems with many tallies. We present one example of the scaling performance: the time per history as a function of the number of cells being tallied.

The problem for this study is a fully reflected iron cube, $64 \mathrm{~cm}$ on a side, with a homogeneous, isotropic Watt energy spectrum source. The problem domain is divided up into individual cells by a 2-cm grid on each axis, for a total of 2097152 unique geometry cells. We tally the energy-integrated flux for a varying number of randomly selected cells in the problem. These scaling results are from a single core of an AMD Opteron ${ }^{\circledR} 6378$ (Piledriver) processor on a single node of Remus, an institutional cluster at Oak Ridge National Laboratory.

Unlike legacy Monte Carlo codes, which use a linear search over tallied cells, Shift uses modern data structures (viz. a hash table) for its implementation, 
so performance shows constant-time scaling with respect to the number of cells being tallied. Figure 2 shows the performance as a function of the number of cells tallied.

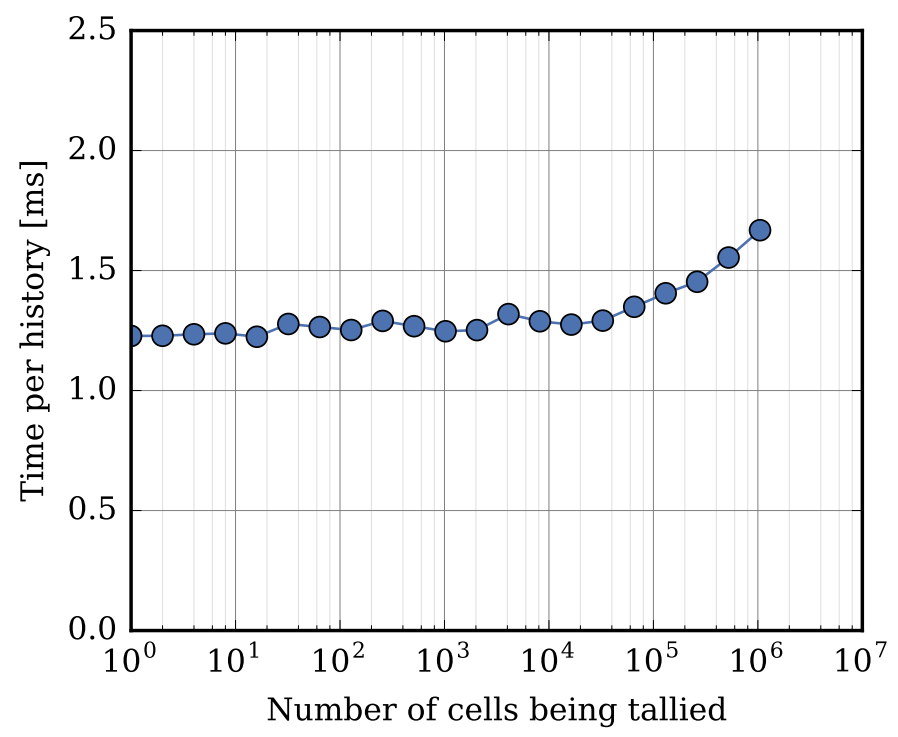

Figure 2: Tally solve time per particle history versus number of tally cells.

For small numbers of cells, the time spent tallying is constant. As the number of tallied cells increases, in this test problem, the number of tally bins encountered in a single history also increases, leading to a linear increase in the solve time. When the problem is changed so that very few cells are encountered in a given history, the number of cells will no longer have an effect on the solution time. We increased the density of the iron cube by a factor of 100 (equivalently, we could have increased the problem and tally cell size while keeping the density constant) and plotted the solution time in Fig. 3.

Several results shown in $\S 3.3$ and $\S 3.4$ show how this tally system scales for a reactor problem run on a supercomputer. Further scaling studies involving the tally system will be performed in a future work. 


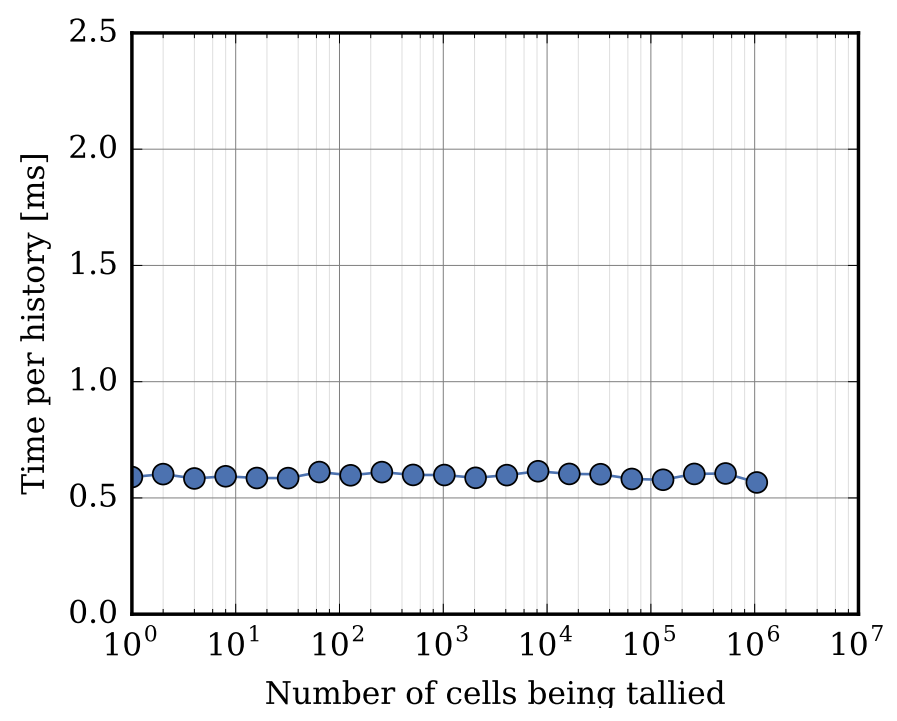

Figure 3: Tally solve time per particle history as a function of the number of tally cells, with a larger mean free path between cells that reduces the number of cells encountered in an average history. 


\subsection{Source Definitions}

Like most other components of Shift, the source routines are written to be modular and extensible. The transport routines are given a Sourcer source manager class that emits particles, and the variety of fixed sources are handled by an additional Source hierarchy (Fig. 4).

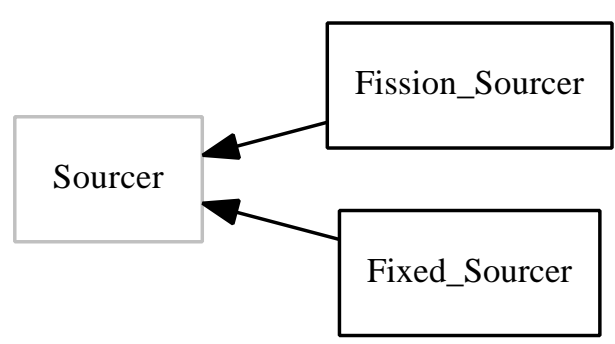

(a) Source managers

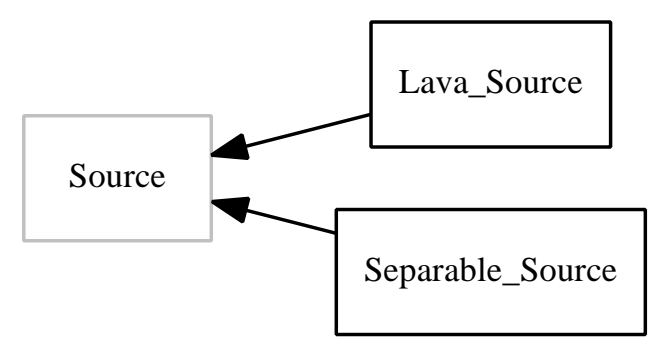

(b) Fixed sources

Figure 4: Inheritance diagram for Shift sources. A Fixed_Sourcer holds one or more Source objects.

\subsubsection{Fixed sources}

Fixed sources in Shift are used both for stand-alone problems and as the initial source distribution for $k$-eigenvalue problems. Shift supports an arbitrary number of source types through the Source abstract class. This class must be able to set a particle's initial state at birth, and also to discretize the source distribution onto a boundary mesh for domain-decomposed problems. Currently, two Source subclasses are available.

The Separable_Source class emits particles in separable distributions of space, energy, and angle. The existing angular distributions include isotropic and monodirectional. Supported energy distributions include histograms (given lower energy bounds and average strength in each bin), line sources (given energies and relative intensities), and a Watt fission spectrum. Currently implemented separable spatial shapes are a point, a rectangular box, a regular cylinder or cylindrical shell, a sphere or spherical shell, and a Cartesian mesh distribution. All spatial distributions are uniform excepting the mesh distribution, which reads a spatial distribution from a Shift HDF5-formatted mesh tally output file.

Separable sources also have the ability to reject source particles in nonfissionable regions: this provides not only a necessary $k$-eigenvalue starting 
source capability but also the ability to perform fixed-source calculations from a fission mesh tally. Fixed-source fission calculations are necessary for coupled neutron-photon physics calculations, and they can also be preferable because weight windows can then be applied meaningfully to obtain lowvariance solutions away from the fissile part of the problem. Additionally, the combination of fission rejection and Cartesian mesh distribution allows a previous eigenvalue calculation's solution to be used as an initial source for a new fission calculation, reducing the number of inactive cycles needed to converge the fission site distribution.

The second Source uses ORNL's Lava library to sample from any userdefined MCNP5 SDEF distribution. Like the MCNP5 geometry support described in $\$ 2.3$, the MCNP5 code generates a runtpe file, which Lava then reads and interprets. It then uses its emulation routines to sample and initialize particles according to the user-specified distribution. Currently, a Lava_Source in Shift can only be used with the corresponding MCNP5 geometry.

\subsubsection{Fission sources}

Shift's fission source uses fission "states" from the prior neutron generation to initialize new fission neutrons. The attributes of the state are determined by the geometry and physics module in use, but they are typically comprised of the spatial location and fission energy of a particle. After the communication of fission sites (see the discussion of Shift's parallel implementation in 2.8), particles are sampled from the stored fission states on each processor. The current fission source implementation is designed to allow straightforward extensions. For example, in the future, we can use fission matrix acceleration to intelligently bias fission site selection.

\subsection{Hybrid Capabilities and Variance Reduction}

In addition to the standard MC variance reduction techniques, Shift has the capability to be used with hybrid variance reduction methods, which are easily enabled in the Exnihilo framework. These hybrid methods can greatly reduce the variance in certain problems and decrease the computational time of the MC calculation [17].

\subsubsection{Standard variance reduction}

By default, Shift uses implicit capture rather than analog tracking. Russian roulette is defined using a weight cutoff $W_{c}$ and survival weight $W_{s}$. If a 
particle has weight $w<W_{c}$ after undergoing a collision, a random number $\xi$ is sampled to determine its survival: if $\xi<w / W_{s}$, the particle lives and has its weight set to $W_{s}$. If $\xi>w / W_{s}$, the particle is killed. This simple algorithm provides a foundation for more complex variance reduction methods such as weight windows.

\subsubsection{Hybrid infrastructure}

Hybrid MC-deterministic methods require that an arbitrary-geometry CE problem be transformed into a spatially discretized MG model for the deterministic method to solve. The Exnihilo framework supports the necessary components for automating the creation of a deterministic problem from a user-given MC problem definition.

The compositions defined by the user's model (MCNP5, KENO, or manually input) use MG cross sections processed by SCALE's XSProc module. Given an AMPX master library (e.g., the 27n19g library with 27 neutron and 19 gamma groups), SCALE runs the infinite homogeneous cross-section processing sequence on each composition. The resulting macroscopic cross sections will, in general, not be accurate enough for a stand-alone solution, but since hybrid methods only require approximate deterministic solutions, the generated cross sections are usually sufficient.

Exnihilo includes a ray tracer that maps Shift geometries onto the structured Cartesian mesh used by Denovo. This ray tracer traces the local mesh on each spatial domain, yielding favorable scaling properties. If a mesh cell contains multiple materials in the continuous model, they are volumetrically mixed inside that mesh cell [18]. The number of rays per cell is adjustable so that users can increase the mixing accuracy at the expense of additional computational time.

Exnihilo additionally uses the ray tracer to map geometry cell tallies and user-specified mesh tallies onto the Denovo mesh. To discretize the separable forward sources in Shift, we implement a generic "discretize" method that takes a Denovo spatial mesh (for spatial distribution) or a set of energy group boundaries (for the energy distribution). By default, both spatial and energy distributions use a parallel point sampling routine. Some spatial distributions (e.g. point collections) and energy distributions (line sources and Watt spectra) are analytically discretized.

Once the approximate deterministic problem is solved, a solution transfer routine communicates fluxes between processors, mapping from the Denovo domain decomposition to the Shift boundary mesh decomposition. This adapter 
enables hybrid calculations to be run on massively parallel architectures because no disk $\mathrm{I} / \mathrm{O}$ is needed.

\subsubsection{Weight windows}

Shift implements spatially decomposed, energy-dependent weight windows. In the traditional form, Shift can use user-assigned weight windows explicitly by reading a field of values from an HDF5 file. Shift also supports direct assignment of weight windows from an adjoint Denovo solution as an importance map.

In both cases, Shift calculates a normalization constant based on the Consistent Adjoint-Driven Importance Sampling (CADIS) methodology [17]. The consistency condition is that a particle born from a biased source must have a weight $w$ equal to the energy-dependent weight window center $\mathrm{w}(\mathbf{r}, E)$ at which it is born. This results in the normalization constant of

$$
R=\iint q(\mathbf{r}, E) \phi^{\dagger}(\mathbf{r}, E) \mathrm{d} E \mathrm{~d} V=\iint \frac{q(\mathbf{r}, E)}{\mathrm{w}(\mathbf{r}, E)} \mathrm{d} E \mathrm{~d} V .
$$

\subsubsection{Biased sources}

During the calculation of the response coefficient, Shift creates biased sources from each of the separable sources in the problem. The separate sources are biased according to their expected response, and the energy and spatial distributions inside each source are also biased.

The separable sources in Shift are represented as

$$
q(\mathbf{x}, E, \boldsymbol{\Omega})=\sum_{s=1}^{S} Q_{s} f(\mathbf{x}) g(E) h(\boldsymbol{\Omega}),
$$

where the distributions $f, g$, and $h$ are normalized by

$$
\begin{gathered}
\int_{V} f(\mathbf{x}) \mathrm{d} V=1, \\
\int_{0}^{\infty} g(E) \mathrm{d} E=1, \\
\int_{4 \pi} h(\Omega) \mathrm{d} \Omega=1 .
\end{gathered}
$$

With these definitions, we construct discretized versions, $f_{i}$ and $g_{g}$, of the spatial and energy components respectively (using the Denovo or user-input 
spatial and energy mesh) and then calculate the response for each source, $s$, by summing the discrete components:

$$
R_{s}=Q_{s} \sum_{i=1}^{I} \sum_{g=1}^{G} f_{i} g_{g} \phi_{n, g}^{\dagger} .
$$

The total response is calculated by summing over these per-source responses,

$$
R=\sum_{S=1}^{S} R_{s},
$$

and each source is biased by the factor $R_{s} / R$, giving higher probability to the sources more likely to contribute to the tally. Each source additionally internally biases its spatial and energy distributions. The total calculated response, $R$, is then used to normalize the weight windows.

A detailed exposition of the hybrid and biased source construction methods used in Shift will be released in an upcoming publication.

\subsection{Parallel Decomposition}

Shift can run on a laptop, but it is a massively parallel code that runs more efficiently on computing clusters and leadership-class machines. In order to run on these machines efficiently, Shift supports multiple parallel decompositions through the Multiple-Set Overlapping Domain (MSOD) algorithm.

\subsubsection{MSOD algorithm}

The MSOD algorithm [19] divides the geometry into structured Cartesian "blocks" based on user-input $x, y$, and $z$ grids. A second user-input parameter is an "overlap" fraction that specifies how much of each block is duplicated between adjacent neighbors in order to reduce the amount of communication needed during the transport solve. The MSOD method replicates this blockdecomposed geometry into "sets" based upon the number of processors

requested. Mathematically, the geometry is decomposed into $N_{\mathrm{s}}$ sets, each of which contains $N_{\mathrm{b}}$ blocks. The number of domains (processes) in a problem is thus

$$
N_{\mathrm{d}}=N_{\mathrm{s}} \times N_{\mathrm{b}} .
$$

The number of particles per set is

$$
N_{\mathrm{p}, \mathrm{s}}=\frac{N_{\mathrm{p}}}{N_{\mathrm{s}}},
$$


where $N_{\mathrm{p}}$ is the number of requested particle histories. An example MSOD problem is illustrated in Fig. 5.

The MSOD setup is determined by a boundary mesh that is used to divide each set into blocks. The number of cells in the boundary mesh is the number of blocks, $N_{\mathrm{b}}$. The boundary mesh is superimposed on the problem geometry; thus no special treatment of the physical geometry is required to implement MSOD. Furthermore, MSOD naturally reduces to full domain replication or full domain decomposition by defining $N_{\mathrm{b}}=1$ or $N_{\mathrm{b}}=N_{\mathrm{d}}$, respectively.

In MSOD there are two principal parallel communication channels: intraset (block-to-block communication within a set) and intra-block (set-to-set communication on the same block). In a $k$-eigenvalue calculation there are three types of communication:

- intra-set communication of particles crossing domain boundaries during transport;

- intra-set and intra-block communication of fission sites between cycles; and

- intra-set and intra-block communication of mesh-based tallies at the end of the problem.

The total number of particles requested by the user is divided among these sets and there is no communication of particles across sets; however, when $N_{\mathrm{b}}>1$, particle histories need to be communicated across blocks, and Shift uses a modified domain decomposition algorithm [20, 21] to handle block-to-block particle communication. The description of this algorithm is given in the following subsection.

For tallies and fission sites, both intra-set communication and intrablock communication are required. Intra-block communication is required to accumulate tally and fission site contributions in overlapping regions to their "parent" blocks; when there is no overlap, no intra-block communication is required for fission sites. However, mesh tallies may require intra-block communication for the case of nonaligned meshes. Because $k$-eigenvalue estimators are global, contributions to $k$ must be performed across all sets and blocks using a full global reduction.

Mesh tally reductions are performed using a two-step process:

1. relay off-block tally contributions to their parent blocks, and

2. perform an intra-set reduction on each block. 


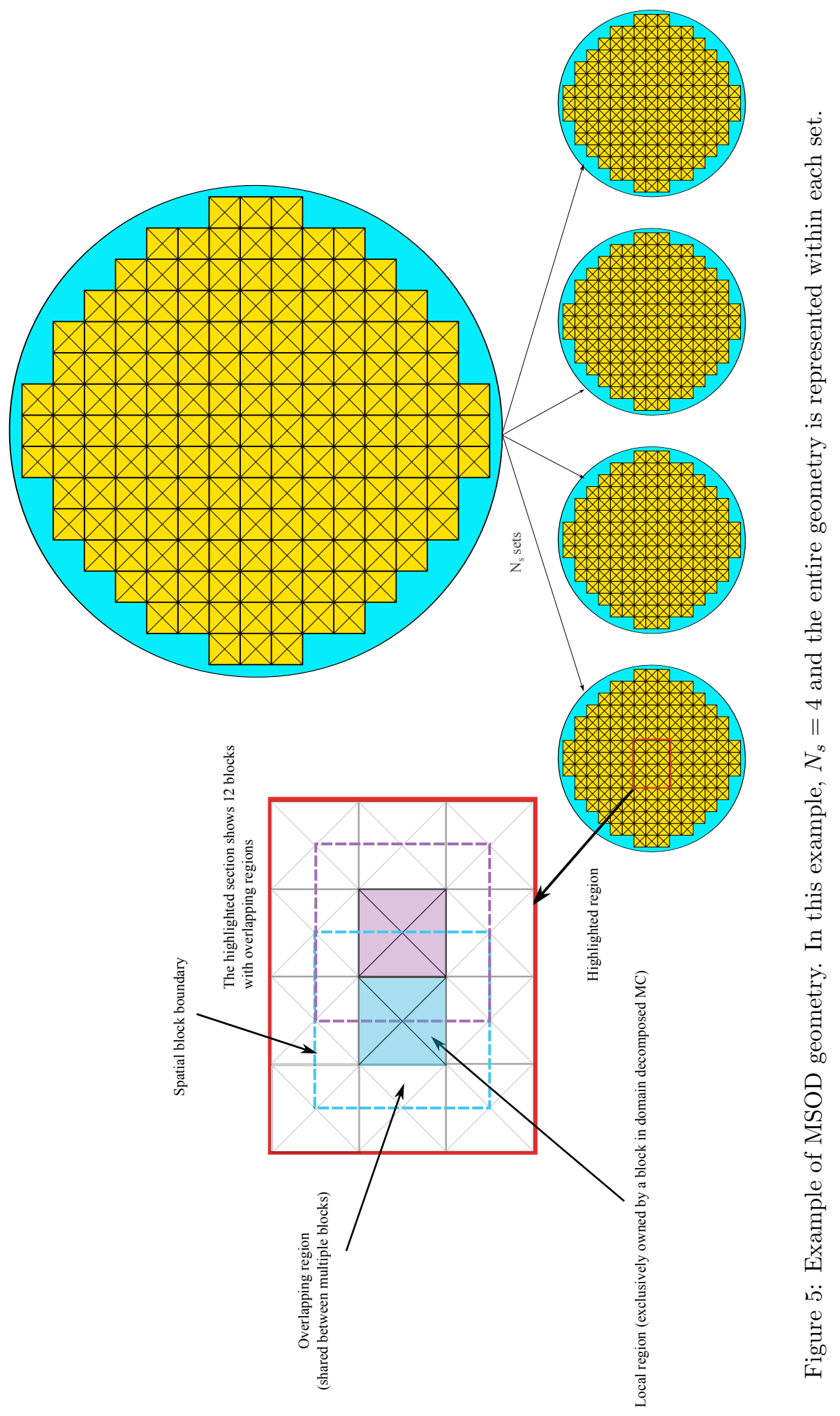


This process is illustrated in Fig. 6. The first step is to form a target mesh on each block. This is a unique decomposition per set in which each tally mesh cell is owned by a block. In the example, tally mesh cell 1 is owned by block 1 on each set, whereas tally mesh cell 4 is owned by block 2 on each set. Next, a source mesh is defined on each block that includes overlapped (shared) tally mesh cells. The overlapped cells can be the result of either a defined overlap region or noncoincident boundary and tally mesh gridlines. Tallies on each block are scored on the source mesh. At the end of the particle transport calculation a global scatter is performed in which tally results are accumulated on each block's target mesh. The global scatter only requires nearest-neighbor communication. Finally, an intra-set global reduction accumulates the global tally results on each block. This reduction is dimensioned by the number of sets.

\subsubsection{Domain-decomposed algorithm description}

As mentioned in the previous section, Shift uses a modified domain decomposition algorithm based upon Refs. [20] and [21] to handle blockto-block particle communication. This algorithm is robust and can handle the communication of particles that have split between blocks. The main difference between the Shift algorithm and the reference algorithm is that all global sums are asynchronous. This asynchronicity removes the race conditions present in the original algorithm. The outline of the solving algorithm in Shift is presented in Alg. 1, details of each method are outlined in Algs. 22-6. 
(a)

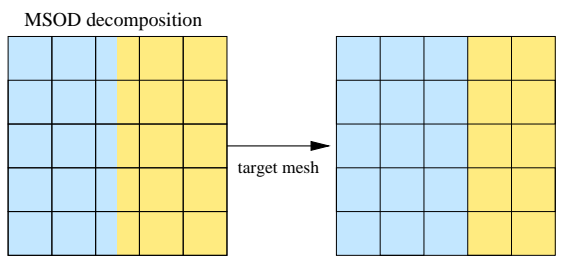

(b)
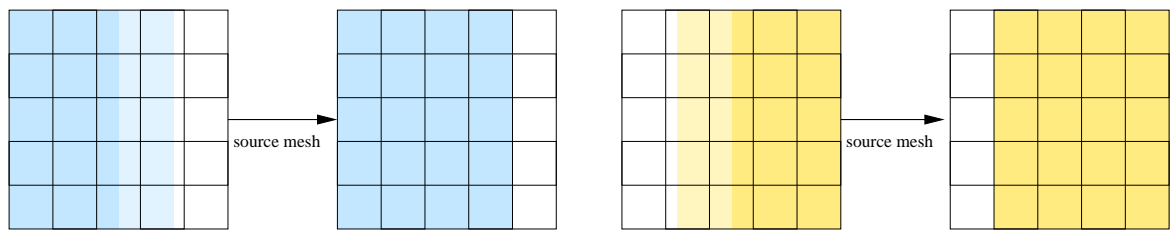

(c)

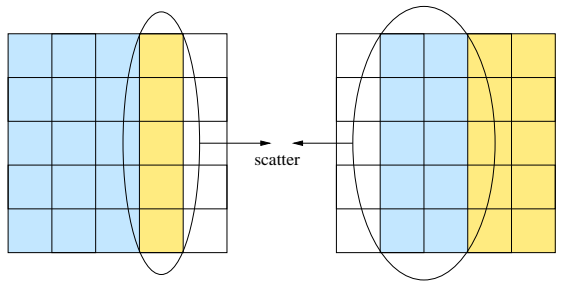

(d)
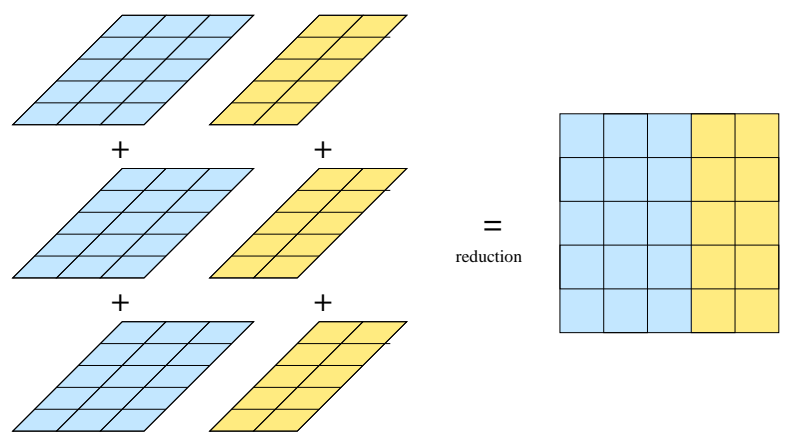

Figure 6: Illustration of a tally reduction with a $5 \times 5$ tally mesh and a $2 \times 1$ boundary mesh yielding 2 blocks per set. In (a) a target mesh is defined for each block. In (b) a source mesh is defined for each block. The boundary mesh overlap regions are indicated by the lightly shaded areas. In (c) a global scatter is performed from the source mesh to the target mesh. In (d) an intra-set reduction is performed to get a final, global tally on each block. 


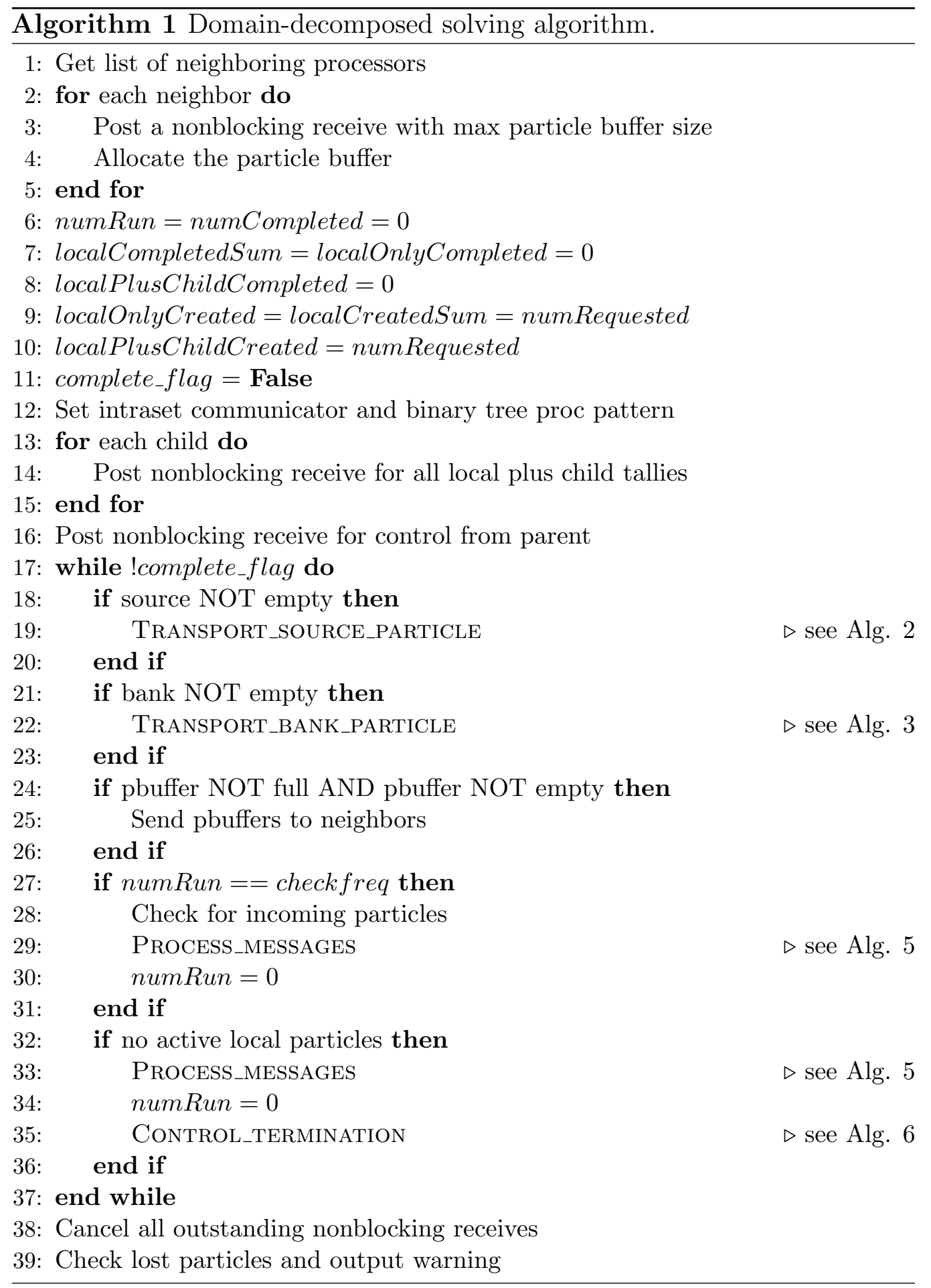



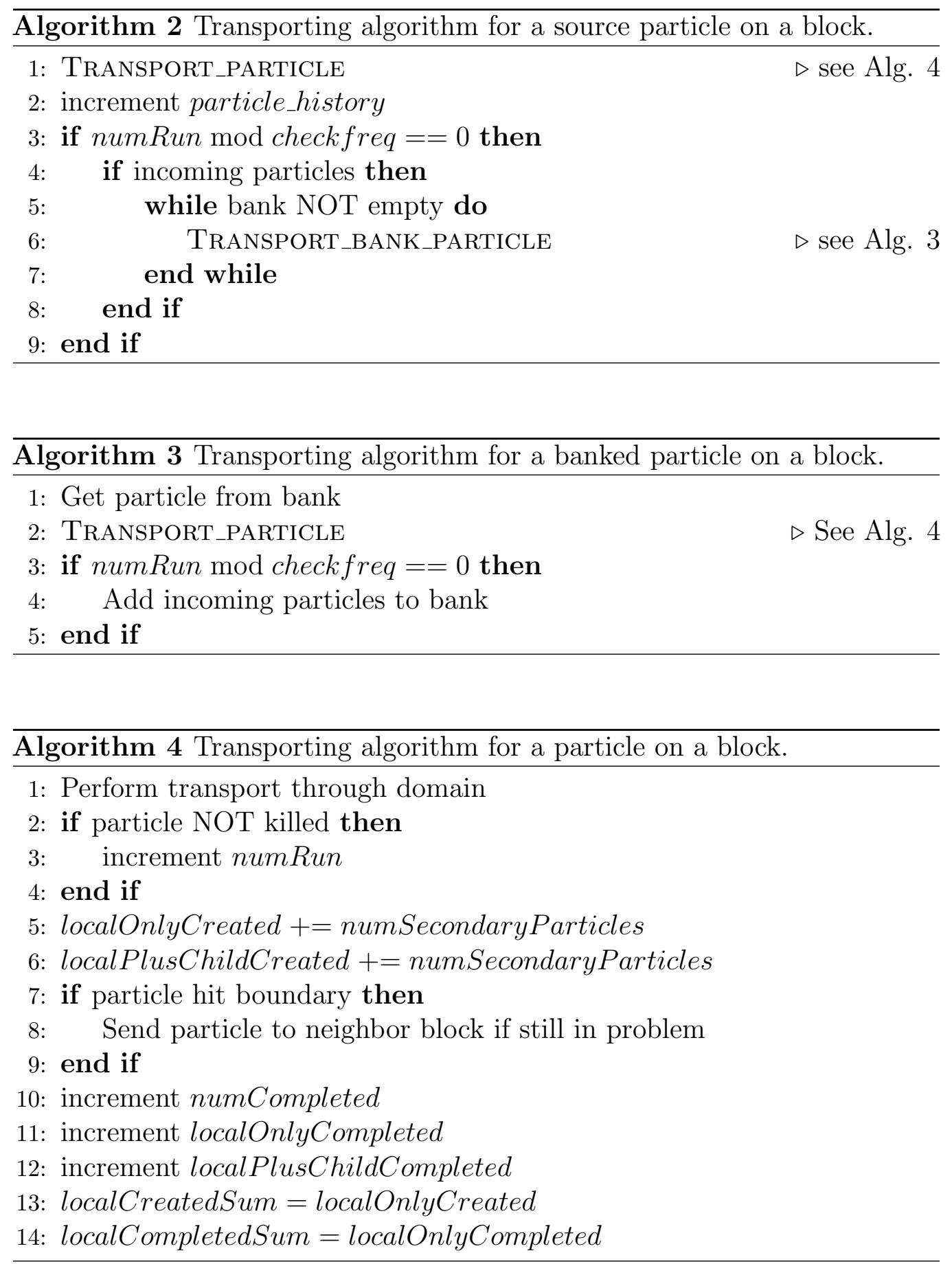


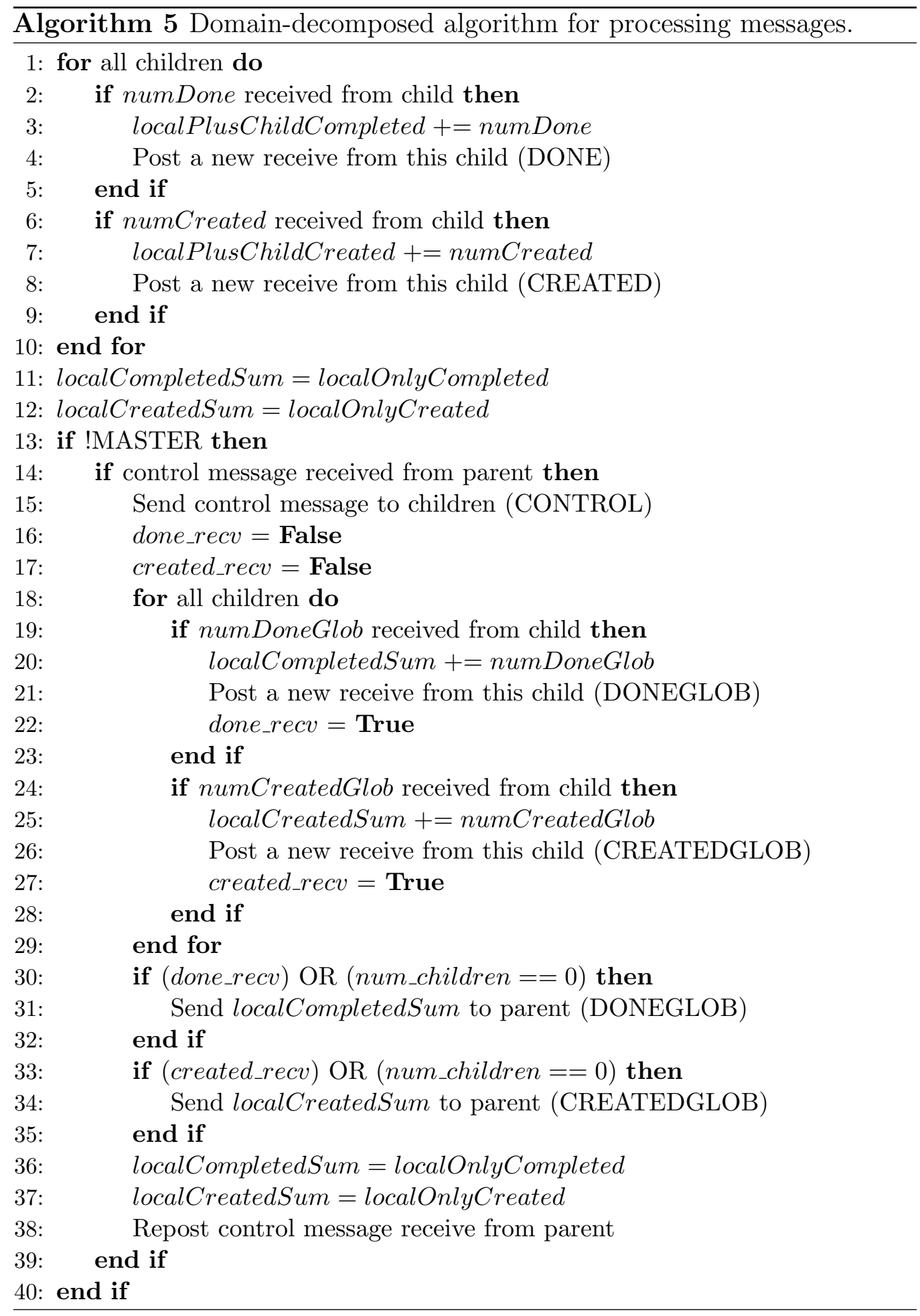




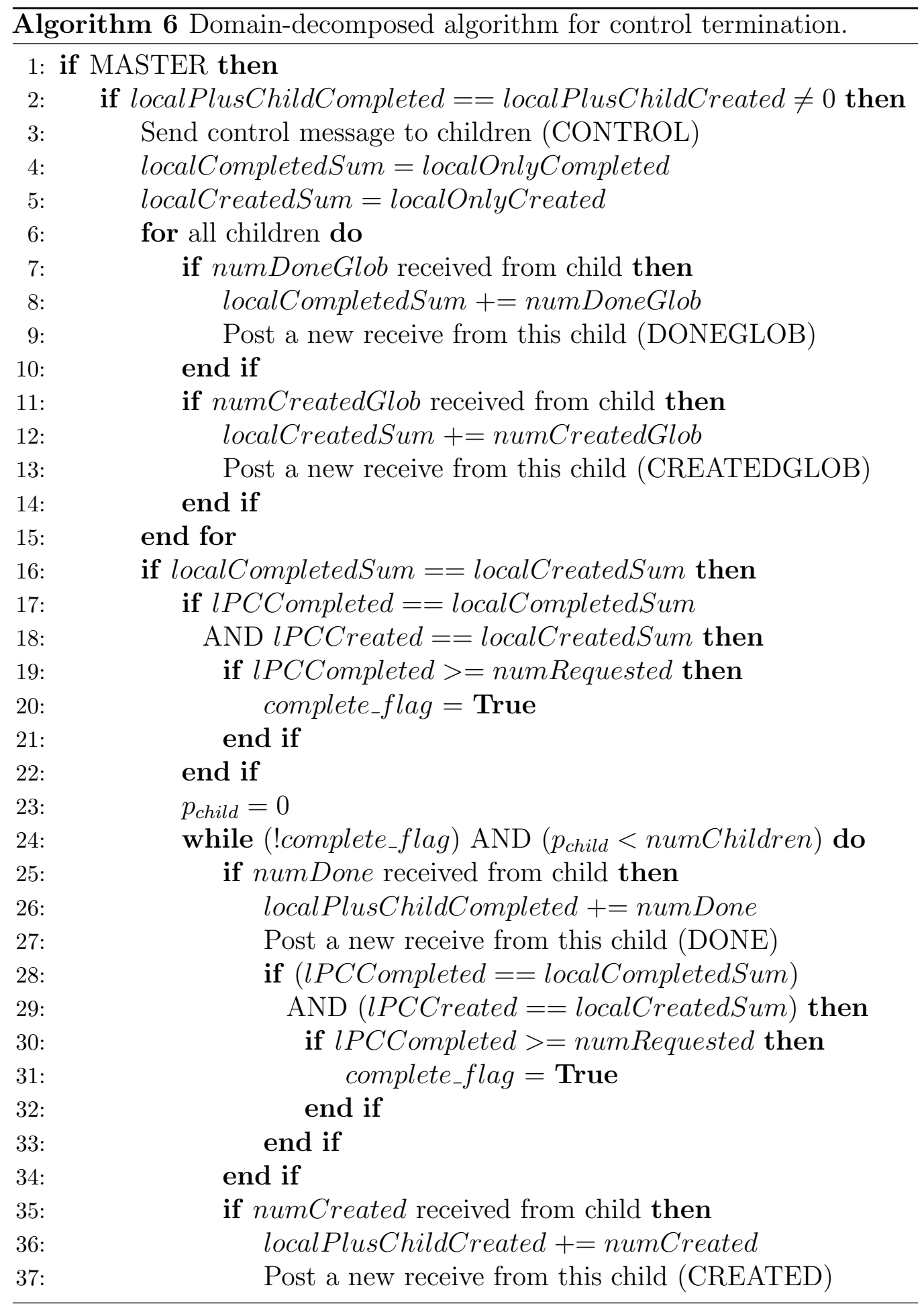




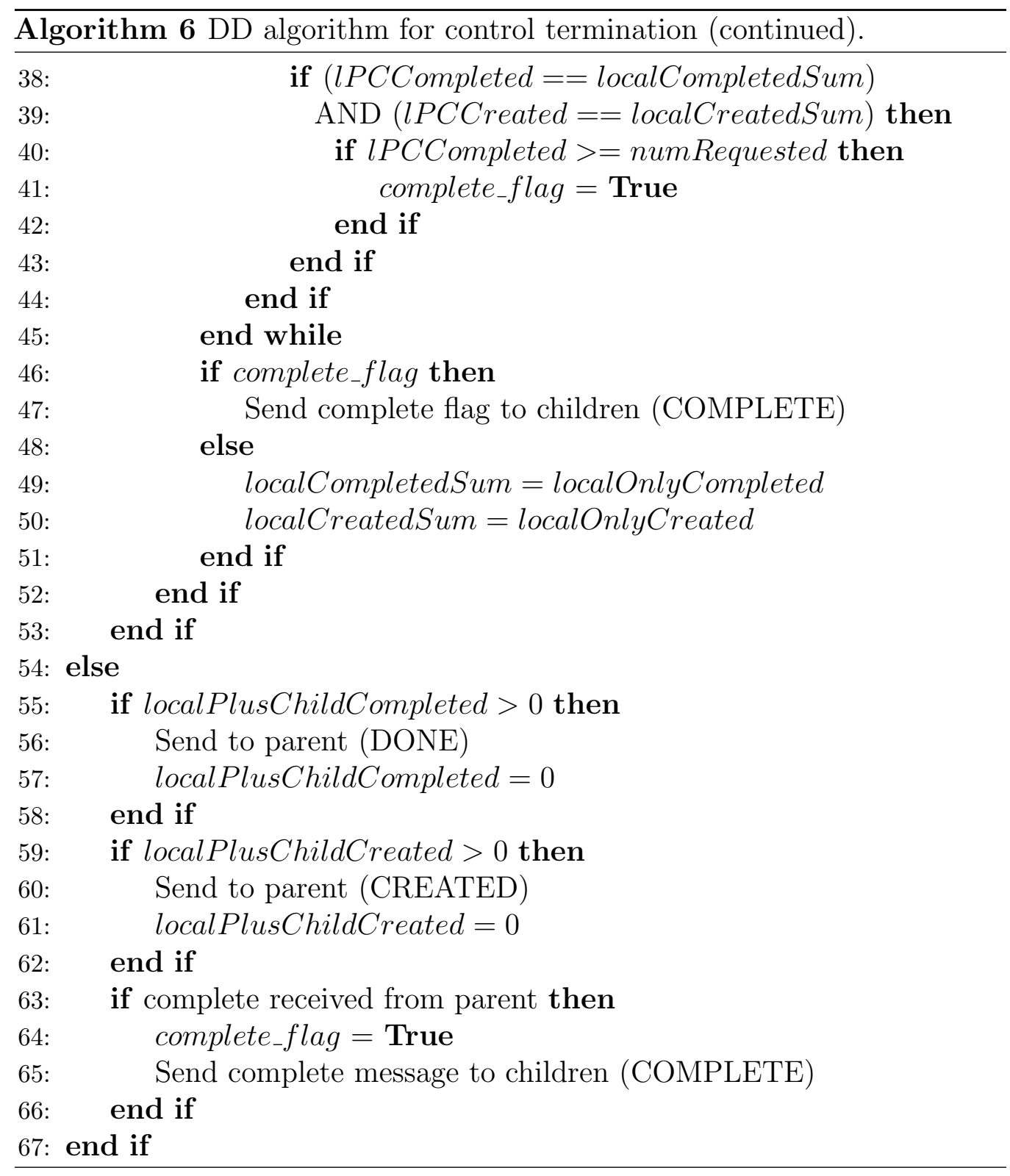




\subsubsection{Fission sites}

In Shift $k$-eigenvalue calculations, the fission source sites for cycle $l+1$ are sampled during the random walk process at each collision site in cycle $l$ according to

$$
n_{\mathrm{f}}=\left[\frac{1}{k_{l}} \frac{\nu \sigma_{\mathrm{f}}}{\sigma_{\mathrm{t}}} w\right],
$$

where $[\cdot]$ is the nearest integer notation, $w$ is the current particle weight, $k_{l}$ is the current estimate of $k_{\text {eff }}$, and $n_{\mathrm{f}}$ is the number of fission neutrons that will be sampled at this location in cycle $l+1$. The resulting fission sites are stored in a fission bank, and these sites constitute the source for the $l+1$ cycle. This sampling statistically preserves the requested total weight of particles, $M$, from cycle to cycle; however, poor estimates of $k_{l}$ can cause large deviations from this value. To account for this statistical deviation, particles in cycle $l+1$ are born with a starting weight of $M / N_{l}$, where $N_{l}$ is the number of fission sites sampled in cycle $l$. This scheme preserves the total weight, $M$, in each cycle.

In a parallel context, this sampling scheme can create load imbalance across sets between subsequent cycles. When a set statistically samples more fission sites than adjoining sets, the fission site population on that set will increase as the iteration progresses. This occurs because the fission site distribution attempts to create $M$ fission sites globally. Thus, after several cycles, all of the particles will be born on a single set. To alleviate this pathology, the fission bank must be rebalanced between cycles. Shift uses an iterative version of the fission bank rebalance algorithm described in Ref. [22] to redistribute the fission sites across sets. This algorithm has demonstrated excellent scalability for full domain replication (one block per set). However, the basic algorithm required modification to handle two specific cases:

1. when sets are out of balance such that nearest neighbors do not have enough sites to send or

2. when there are multiple blocks per set (MSOD).

To alleviate the first condition, the original algorithm is wrapped in an iteration scheme until full fission bank balance is achieved across all sets. To handle multiple blocks, an additional constraint must be applied that limits set-to-set transfers based on similar block populations of fission sites.

We now describe the fission bank rebalance scheme employed in Shift. 
(a)

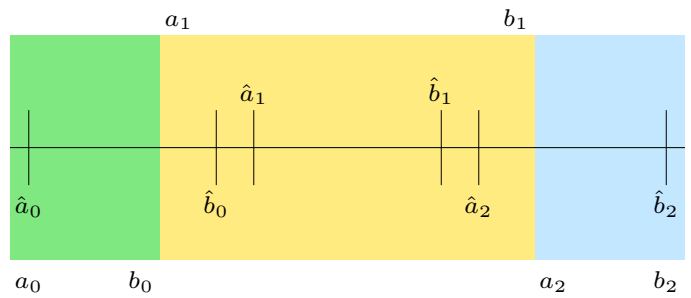

(b)

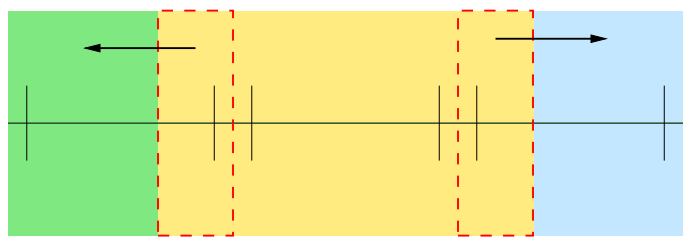

(c)

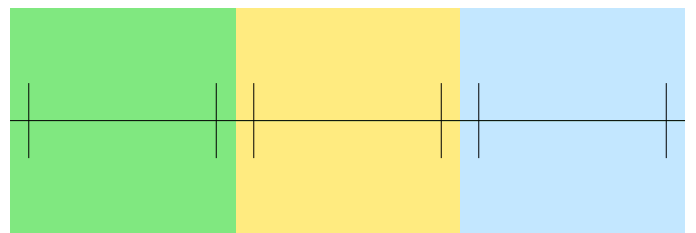

Figure 7: Rebalance boundaries across sets.

The objective is to place the same number of fission sites on each set using

$$
n_{i}= \begin{cases}\left\lfloor\frac{N_{l}}{N_{\mathrm{s}}}\right\rfloor+1 & N_{l} \bmod N_{\mathrm{s}}>0, \\ \left\lfloor\frac{N_{l}}{N_{\mathrm{s}}}\right\rfloor & N_{l} \bmod N_{\mathrm{s}}=0,\end{cases}
$$

where $n_{i}$ is the desired number of fission sites on set $i$. If the global number of fission sites are monotonically ordered over the range $\left[0, N_{l}\right]$, the desired bounds $\left(\hat{a}_{i}, \hat{b}_{i}\right)$ on each set can be defined by the recurrence relation

$$
\begin{aligned}
\hat{a}_{0} & =0, \\
\hat{b}_{i} & =\hat{a}_{i}+n_{i}-1, \\
\hat{a}_{i+1} & =\hat{b}_{i}+1, \quad i=0, \ldots, N_{\mathrm{s}} .
\end{aligned}
$$

The actual bounds of the unbalanced fission bank on each set is given by $\left(a_{i}, b_{i}\right)$ as illustrated in Fig. 7(a). 
The goal is to align the actual fission bank boundaries on each set with the target boundaries. Thus, on each set we apply Alg. 7, in which the actual fission bank boundaries are compared to the target boundaries and any surplus is communicated to the left-neighbor set $(i-1)$ or right-neighbor set $(i+1)$ as shown in Fig. 7(b).

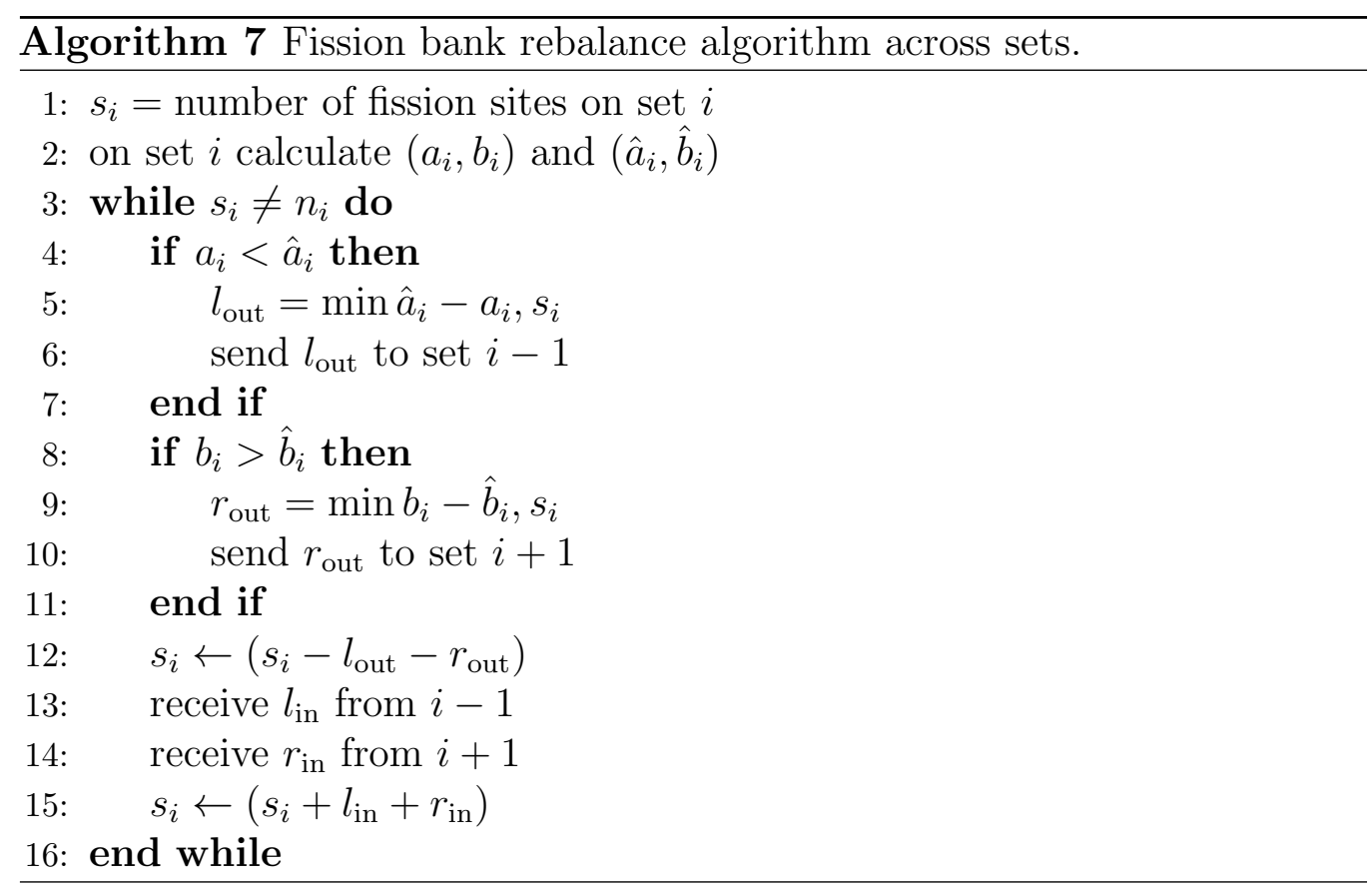

As long as fission sites received from the left and right neighbors are prepended and appended, respectively, to the local fission bank, the global ordering of the fission bank will not change; see Fig. 7(c). Thus, for fully domain-replicated problems $\left(N_{\mathrm{b}}=1\right)$, reproducibility can be preserved regardless of the size of $N_{\mathrm{s}}$. Furthermore, since each set is only required to transfer sites with its left and right neighbors, the communication cost is local.

When the number of blocks per set is greater than 1, the fission site rebalance must take into account the block structure within a set. Communication between sets is constrained to equivalent blocks, as shown in Fig 8 .

The number of sites that get passed between sets is determined by Alg. 7 ; furthermore, communication is constrained between equivalent blocks. The degree of freedom in Alg. 7 is the number of sites to pull from each block. For reactor problems, we desire equal numbers of particles on each block within a 


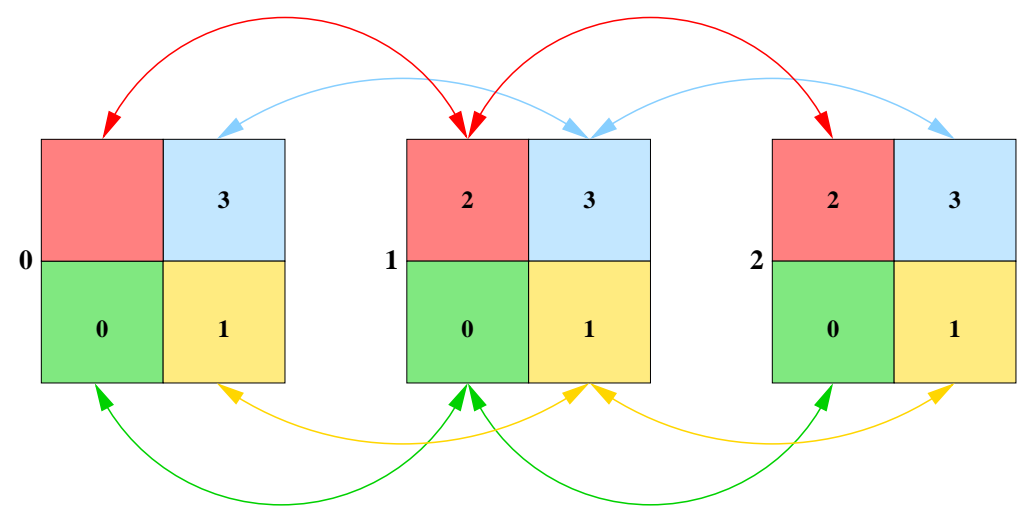

Figure 8: Illustration of block-to-block communication between sets during fission bank rebalance. Communication is constrained to equivalent blocks in adjacent sets.

set to achieve ideal load balancing. This condition may not hold for other types of problems that have large nonfissionable regions; however, the blocks for a reactor problem can generally be defined such that each block has a fission source of similar magnitude.

To preserve load balance, Shift calculates the average number of fission sites per block that is in each set, $\bar{n}_{\mathrm{b}}$. The number of surplus sites is calculated on each block, $s_{\mathrm{b}}=n_{\mathrm{b}}-\bar{n}_{\mathrm{b}}$, where $n_{\mathrm{b}}$ is the number of sites on the block. Fission sites are communicated from this surplus by pulling sites uniformly from each block. If there are not enough surplus sites, regular sites are chosen uniformly from each block.

\subsubsection{MSOD scaling study}

We performed a small scaling study of the MSOD and fission site rebalance algorithms using a simple reactor eigenvalue problem. The reactor problem consists of a water-moderated $4 \times 4$ assembly configuration with each assembly made of a $17 \times 17$ array of $\mathrm{UO}_{2}$ fuel pins. This problem uses SCE physics and RTK geometry. The decomposition is fixed as a $2 \times 2$ array of blocks. The strong and weak scaling studies were performed on the OLCF machine, Titan [23].

Strong scaling was simulated by keeping the number of particle histories in the full problem constant as the number of cores was increased; i.e., the number of particle histories per set decreased with increasing core count. The total number of particle histories was $5 \times 10^{6}$. Figure 9 shows the efficiency 


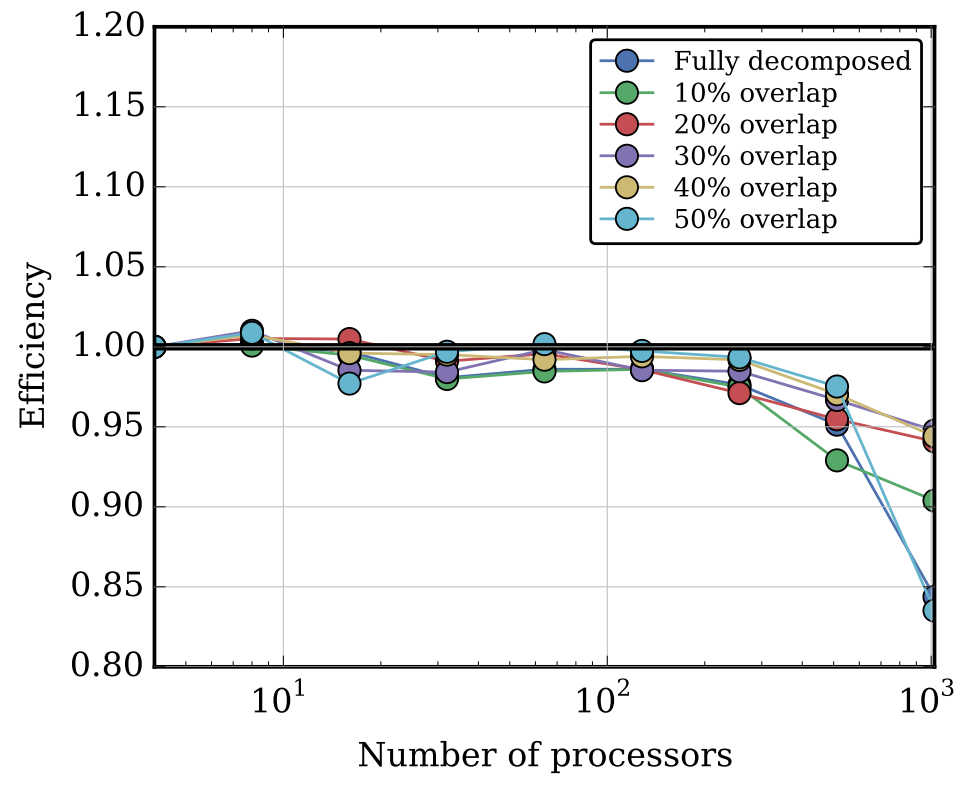

Figure 9: Strong scaling of the $4 \times 4$ reactor assembly problem. The plot shows efficiency versus the number of processors. Each set is decomposed into $2 \times 2$ blocks.

achieved per set with a $2 \times 2$ decomposition per set on an internal cluster. We see from Fig. 9 that all of the overlap fractions yield very similar scaling results for this problem. All of these overlap fractions give good strong scaling over sets with an efficiency ranging from $100 \%$ down to $84 \%$.

A weak scaling study was also performed using the same problem. Weak scaling was simulated by keeping the number of particle histories per set constant at $6 \times 10^{4}$ and by increasing the total number of particle histories in the problem with an increasing number of sets. Figure 10 shows the excellent weak scaling of the MSOD algorithm on Titan for this problem. The parallel efficiency for various overlap fractions is very close to the ideal scaling.

\subsection{Depletion Package}

Ongoing work is being performed to enable coupling between Shift and ORIGEN, a depletion/transmutation analysis code, using ORIGEN's new $\mathrm{C}++$ API [7]. Unlike previous codes that attempted to couple stand-alone MC and ORIGEN through scripts and file-based I/O (e.g., VESTA [24], Monteburns [25]), Shift uses a new in-memory C++-based API in ORIGEN. 


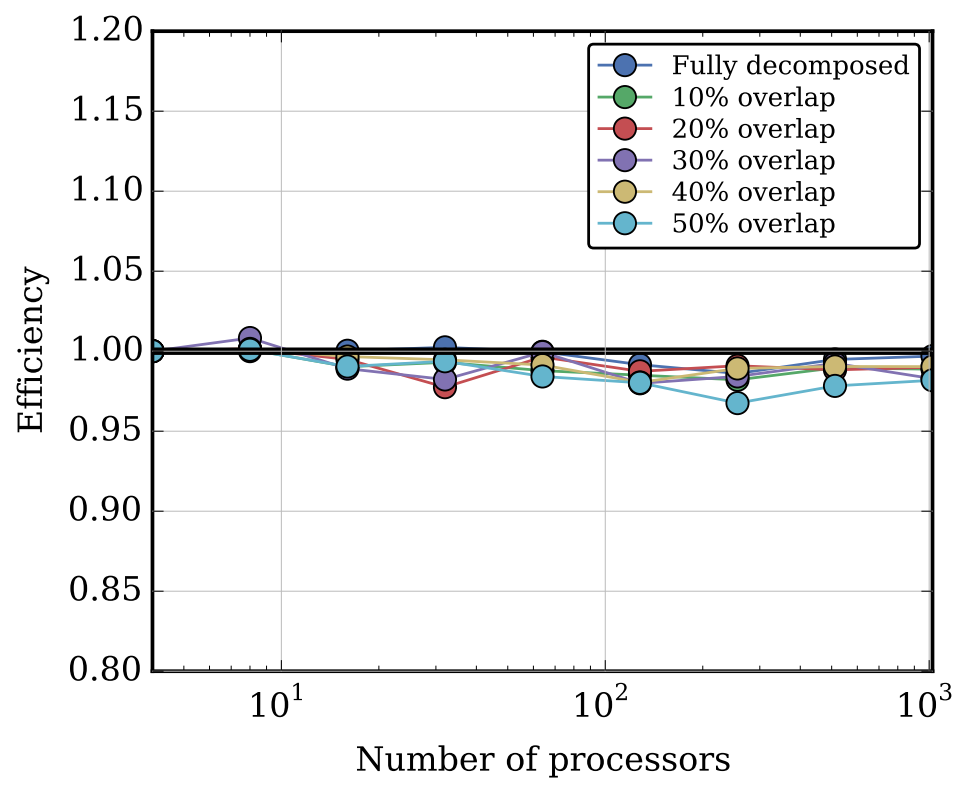

Figure 10: Weak scaling of the $4 \times 4$ reactor assembly problem with each set decomposed into $2 \times 2$ blocks. The plot shows efficiency on Titan versus number of processors. 
This avoids the prohibitive cost (on HPC systems) of writing to disk between transport and depletion steps and enables depletion to be performed on each depletable region in parallel.

To obtain the microscopic per-nuclide reaction rates needed for depletion, Shift currently uses the same approach as VESTA [24], tallying ultrafine-group fluxes in each depletion region, then using these fluxes to collapse microscopic $\mathrm{CE}$ cross sections into one-group reaction rates. These reaction rates are then provided to ORIGEN for the depletion calculation.

Currently, Shift implements constant-power depletion using either a forward Euler or a "middlestep" approach (similar to an option available in VESTA). The middlestep approach operates by first calculating the fluxes, $\phi_{n}=\phi\left(t_{n}\right)$, at the beginning of the time step. The fluxes are then used to deplete the initial nuclide concentrations, $C_{n}=C\left(t_{n}\right)$, to their value at the middle of the time step, $C_{n+1 / 2}=C\left(t_{n+\frac{1}{2}}\right)$, where

$$
t_{n+1 / 2}=\frac{1}{2}\left(t_{n}+t_{n+1}\right) .
$$

A transport calculation is then performed to obtain an estimate of the timestep-averaged fluxes:

$$
\tilde{\phi}_{n+1 / 2}=\tilde{\phi}\left(t_{n+\frac{1}{2}}\right) \approx \frac{1}{t_{n+1}-t_{n}} \int_{t_{n}}^{t_{n+1}} \phi(t) \mathrm{d} t .
$$

This midpoint estimate of the flux is used to calculate the reaction rates and deplete the initial concentrations, $C_{n}$, to their end-of-time-step value, $C_{n+1}$. The middle-of-step nuclide concentrations, $C_{n+1 / 2}$, are only used to calculate a more accurate estimate of the time-averaged fluxes. The accuracy of this middlestep method has recently been investigated [26, 27]. These studies show that the accuracy of this method can be adequate for many problems. Higher-order methods are currently under development and investigation.

To optimize the parallel efficiency of the depletion calculation, Shift distributes the depletable regions on each block among the available processors. Thus it minimizes the number of depletion solves performed on each core. After every core has calculated the new concentrations for its depletion regions, the results are broadcast to every other core on the block.

In Alg. 8 we summarize the depletion algorithm used in Shift for a single depletion solve. Shift writes the isotopic results and burn data for each depletion step to an HDF5 file. In the future, we intend to use this HDF5 file for a depletion restart capability. This package is currently undergoing 
benchmarking against experiments and other coupled transport-depletion codes.

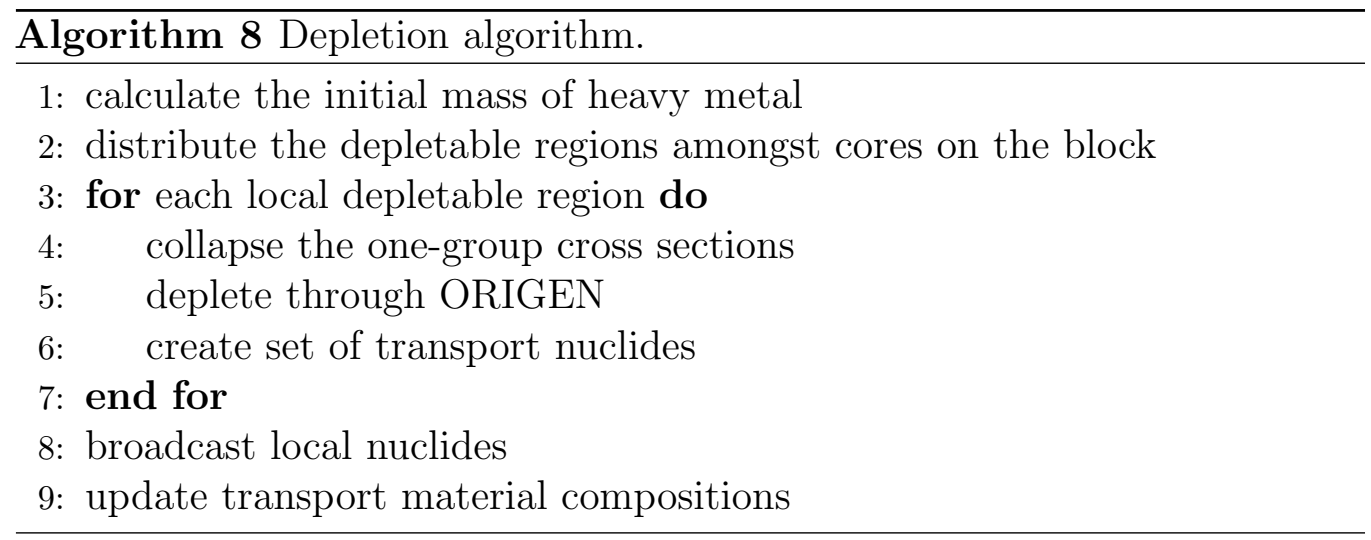

\section{Validation and Verification}

A variety of testing and benchmarking problems have been run through Shift for verification and validation. In this section we focus on LWR reactor physics tests that have been performed. A selection of results presented here includes an overview of the small reactor core benchmark from Babcock and Wilcox (BW1810), two CASL VERA benchmark problems, Westinghouse AP1000 ${ }^{\circledR}$ PWR simulations, and the NEA MC Performance Benchmark [28]. Overall, we show that Shift performs very well on these problems, which include experimental and other MC code results, while being able to take advantage of leadership-class computing machines. Testing and benchmarking against other reactor types are currently ongoing.

\subsection{BW1810 Benchmarks}

The BW1810 criticality experiments [29] provide a valuable benchmark for neutronics calculations of nuclear reactors. This benchmark includes small LWR experiments to investigate pin powers, the effect of reactor configurations, and reactivity worths for various control rods. In this study, we compare eigenvalues, pin powers, and reactivity rod worths calculated using Shift to published data from the benchmark experiments.

The full specifications for these reactor cores are given in the benchmark report [29]. An example of one of the BW1810 core configurations is shown in Fig. 11. The figure shows a slice in the $x y$ plane of BW1810 core XIII to 
give an idea of the core layouts. Fuel pins are shown in red and green, the moderator is shown in light blue, control rods are shown in purple, and the vessel is shown in dark blue. Variations of this core layout were simulated in Shift to correspond with the experiments in the benchmark [29].

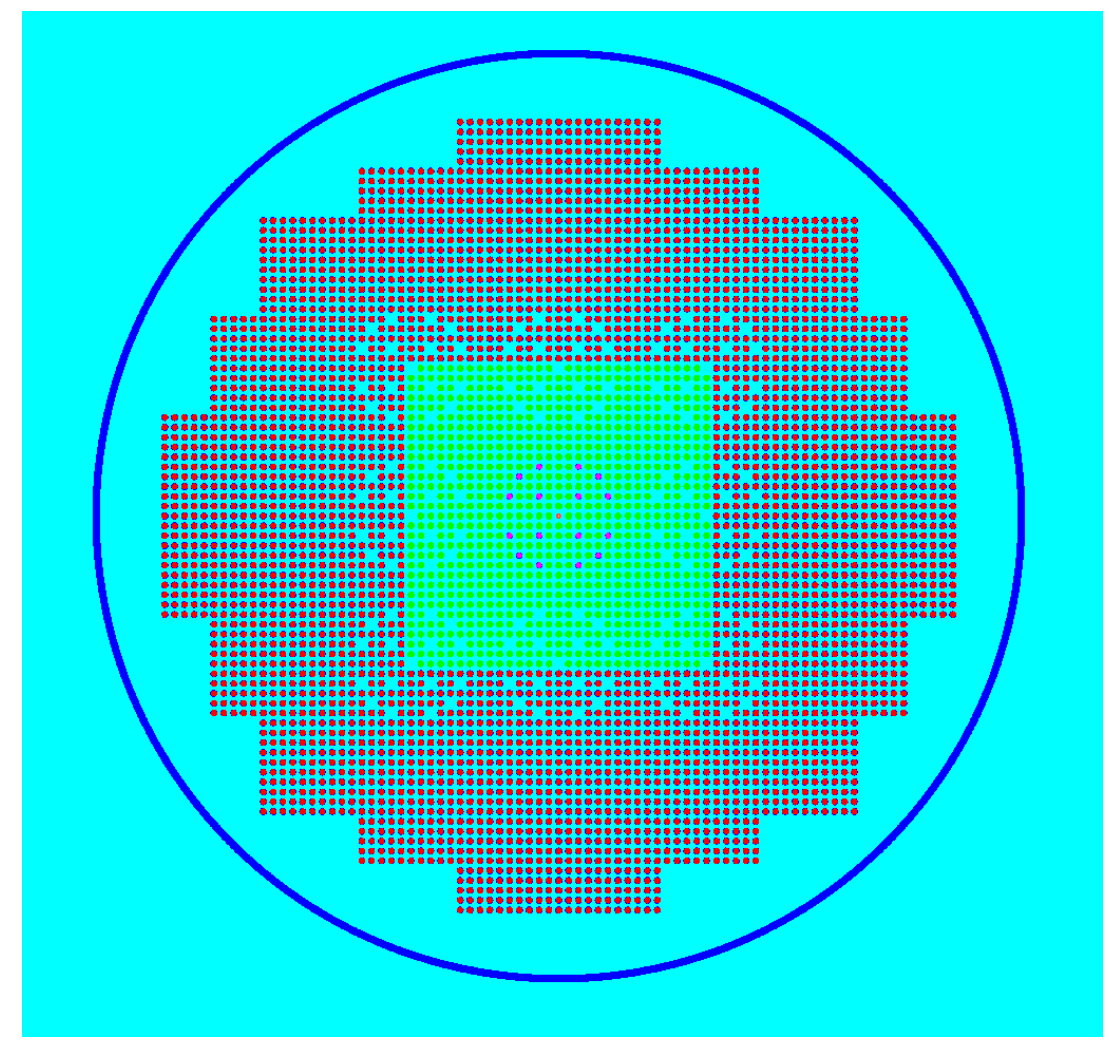

Figure 11: BW1810 core-XIII materials in $x y$ slice of core layout.

Cores I through XX, excluding core XI, were modeled and simulated using Shiftand these results were compared to the benchmark results. Shift did not model any structures past the reactor vessel and did not model the center grid plate nor the top grid plate. These simulations used ENDF-VII.0 data processed using the SCALE AMPX module [14].

These models are based on critical experiments. Therefore, the simulated $k_{\text {eff }}$ values are compared with a $k_{\text {eff }}$ of unity. The average, minimum, and maximum reactivity differences over all simulated core configurations were 42 , -117 , and $-47 \mathrm{pcm}$, respectively. Shift produces eigenvalue results very close to the benchmark. A difference of less than $100 \mathrm{pcm}$ with the benchmark 
eigenvalue is considered good for these experiments. Of course, $k_{\text {eff }}$ is not an adequate measure of benchmarking for these small reactor problems, so we also look at rod worths and pin powers.

An approximate calculation of the value of boron parts per million (ppm) per pcm $\left(1 \times 10^{-5}\right)$ of reactivity was performed using Shift simulations of three of the BW1810 cores. Then, we used this value to calculate the Shift rod worth values and compared them with the benchmark values. Figure 12 shows the percent error in $\mathrm{B}_{4} \mathrm{C}$ and gadolinium $(\mathrm{Gd})$ fuel rod worths approximated using Shift for various cores as compared to the benchmark values. Shift approximates the $\mathrm{B}_{4} \mathrm{C}$ rod worths to within $1.5 \%$ of the benchmark and the Gd fuel rod worth to within $6 \%$.

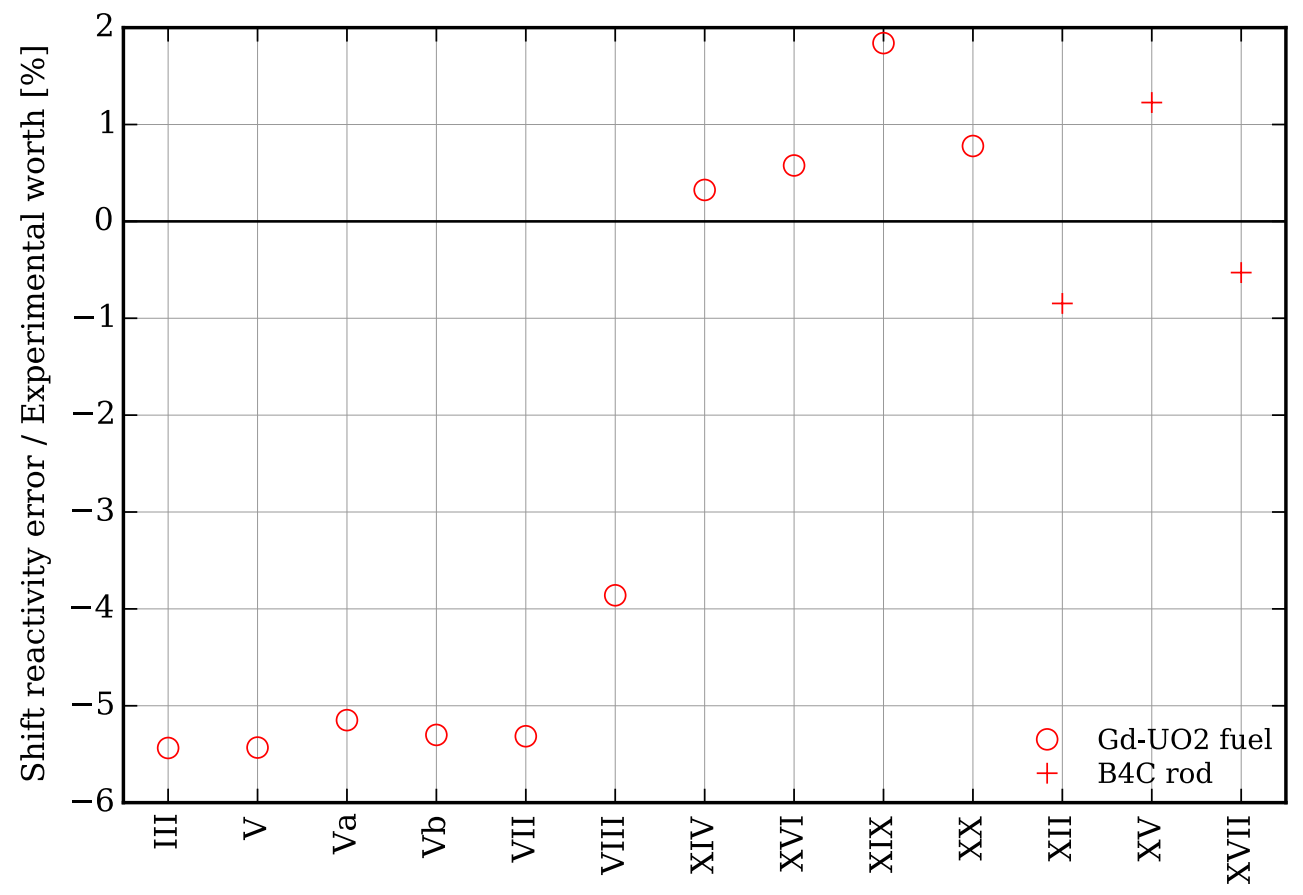

Figure 12: Absolute error of Shift simulations of $\mathrm{Gd}-\mathrm{UO}_{2}$ fuel and $\mathrm{B}_{4} \mathrm{C}$ rod worths from the BW1810 experiment. 
Next, we show pin power comparisons between Shift and the benchmark experiments. These pin powers are at the midplane of the center assembly as reported in the benchmark. We define the relative difference in the following figures as

$$
\text { Rel. Diff. } \equiv \frac{\text { Experiment Pin Power }- \text { Shift Pin Power }}{\text { Experiment Pin Power }}
$$

Cells shown in black in these figures are either water holes, control rods, or instrument tubes according to the core layouts defined in the benchmark. Figure 13 shows the relative difference between Shift and the measurements for normalized pin powers in the center assembly at the midplane for core I. These results give an RMS $=0.6 \%$ and a maximum error of $1.4 \%$, indicating very good agreement, less than $2 \%$, with measured data. Figures 14 through 18 show the relative differences in pin powers between Shift and the benchmark for various cores. All of these have RMS differences around or below $2 \%$. The larger maximum relative error in pin powers for cores XVIII and XX occurs near the center water hole.

A future paper will cover the complete details of this Babcock and Wilcox benchmarking study. Overall, these BW1810 results have shown that Shift solutions can accurately reproduce experimental data. 


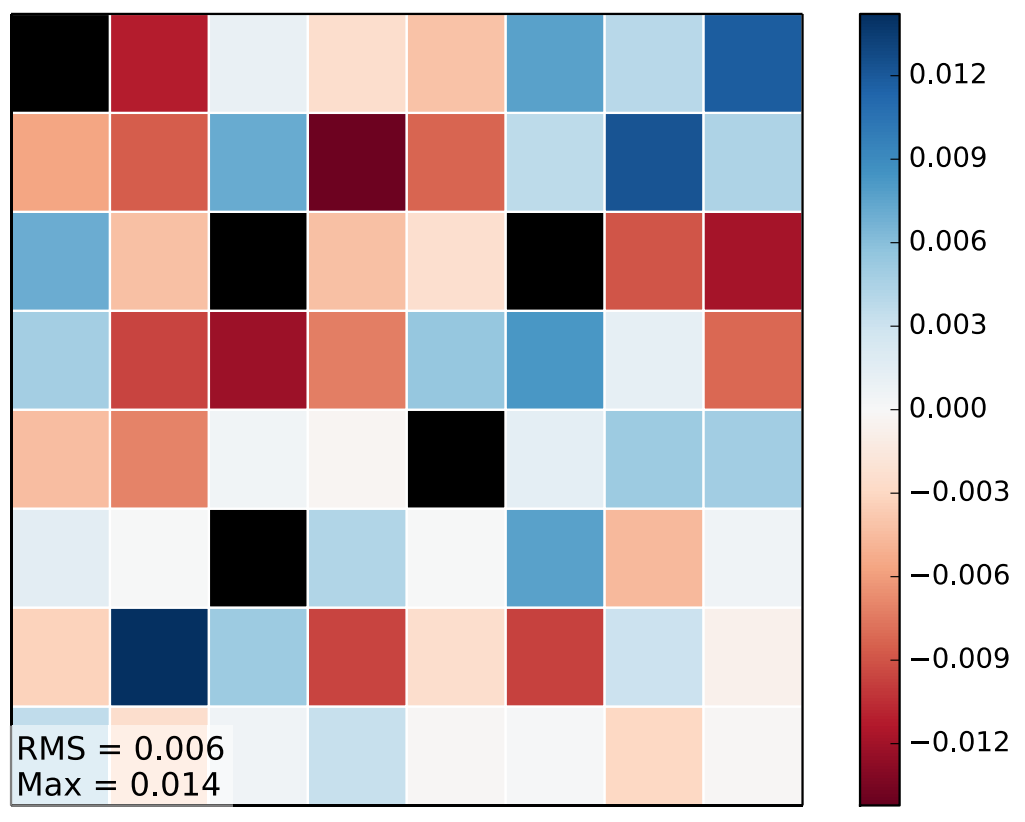

Figure 13: BW1810 core-I relative difference in midplane pin powers for a quarter of the center assembly between Shift and the benchmark. 

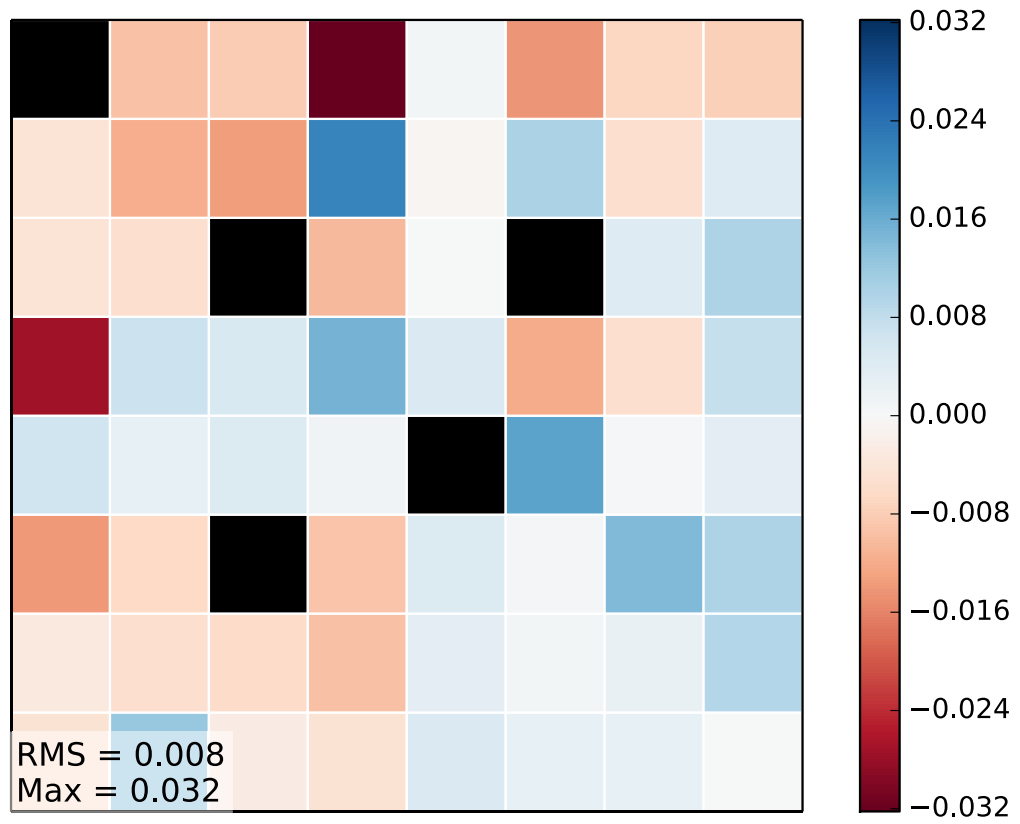

Figure 14: BW1810 core- $\mathrm{V}$ relative difference in midplane pin powers for a quarter of the center assembly between Shift and the benchmark. 


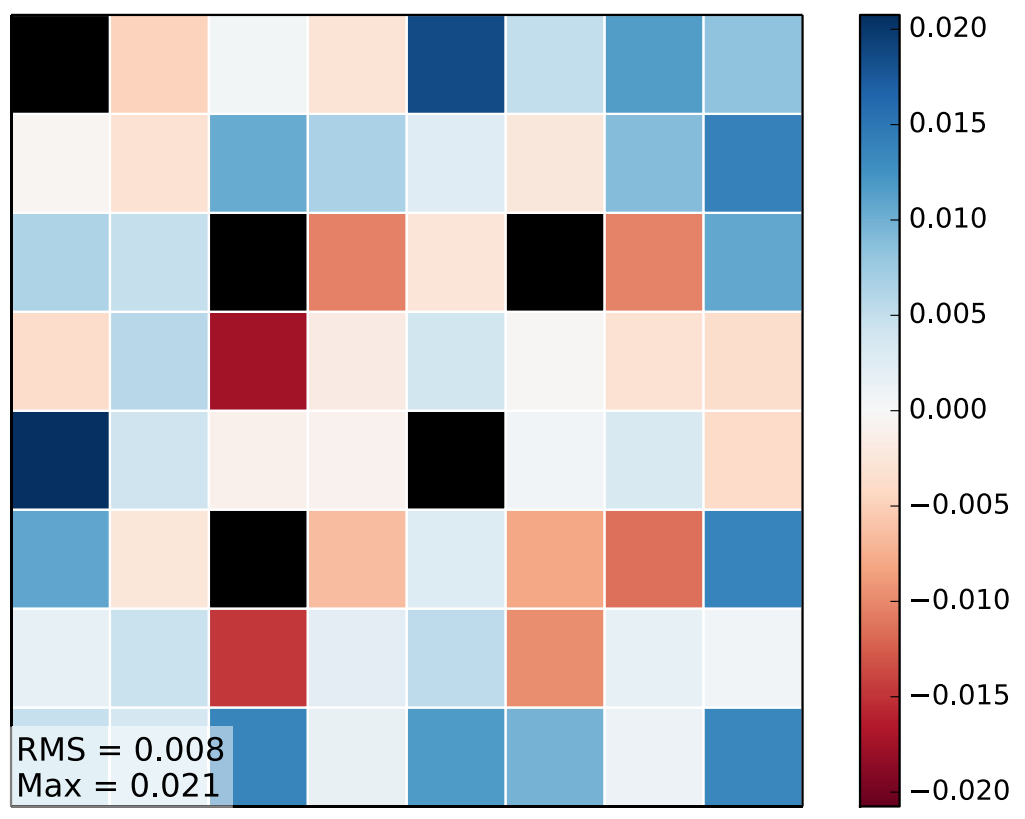

Figure 15: BW1810 core-XII relative difference in midplane pin powers for a quarter of the center assembly at the midplane between Shift and the benchmark. 

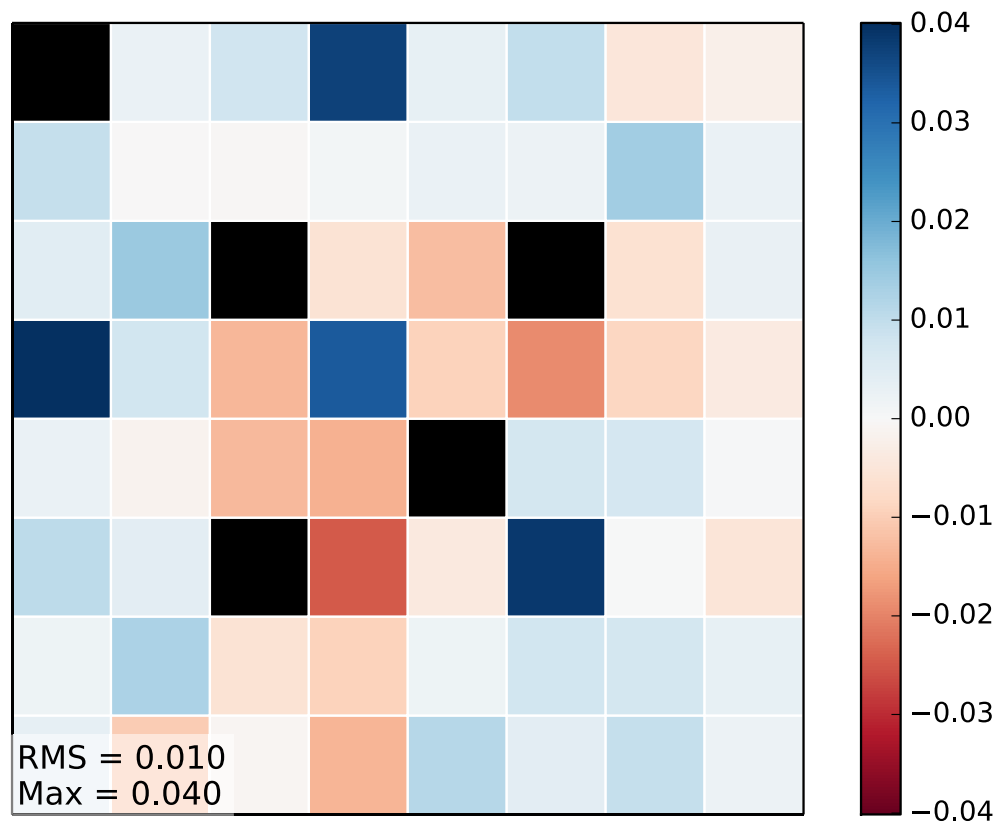

Figure 16: BW1810 core-XIV relative difference in midplane pin powers for a quarter of the center assembly at the midplane between Shift and the benchmark. 


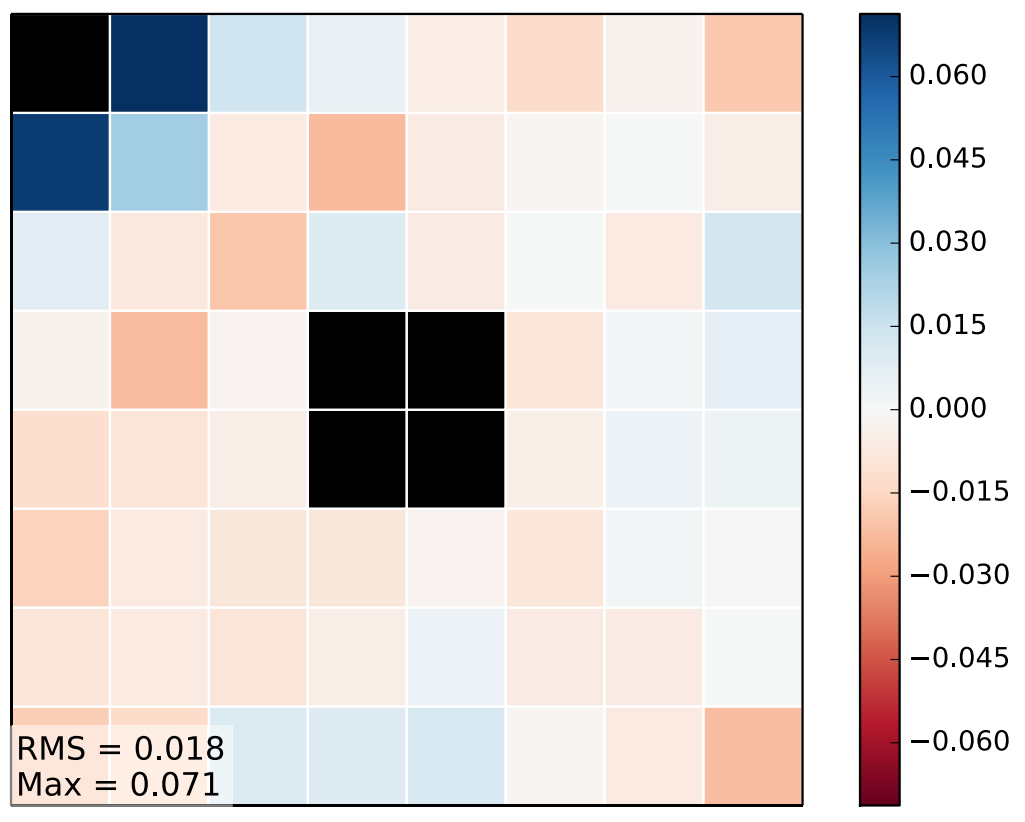

Figure 17: BW1810 core-XVIII relative difference in midplane pin powers for a quarter of the center assembly at the midplane between Shift and the benchmark. 


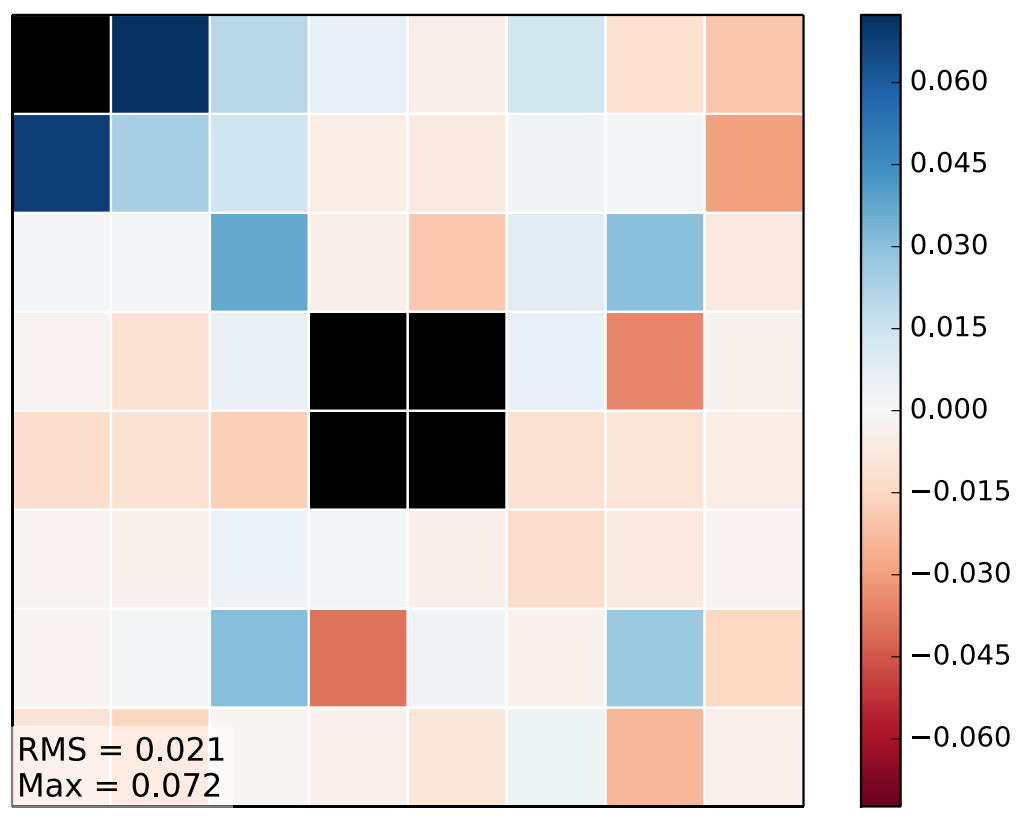

Figure 18: BW1810 core-XX relative difference in midplane pin powers for a quarter of the center assembly at the midplane between Shift and the benchmark. 


\subsection{CASL VERA Benchmarks}

The CASL VERA test suite contains a set of benchmark problems run for Shift validation [30. These benchmarks used the SCALE CE KENO-VI code to produce accepted solutions [7. They were originally designed to be compared with deterministic transport code simulations, but this paper uses these MC results for Shift benchmarking. The full problem specifications can be found in Ref. [30]. In this paper, we show comparison results for problems 2 and 5 . All of the results shown for these problems were produced from runs on the OLCF supercomputer, Titan [23].

\subsubsection{VERA problem 2}

VERA problem 2 is a two-dimensional array of fuel rods typical of the central axial region of PWR fuel assemblies based on a Westinghouse-type $17 \times 17$ assembly at beginning-of-life (BOL). The purpose of this benchmark is to predict the eigenvalue and pin power distribution in the assembly. The reference solution and full details of the problem specification can be found in Ref. [30].

Variations of this two-dimensional assembly were run (17 assemblies total) using Shift via the Insilico front end. Each calculation was run on Titan using 1100 total cycles with 100 inactive cycles and $1 \times 10^{6}$ particle histories per cycle. Table 1 shows the comparison of $k_{\text {eff }}$ and fission rate densities between Shift and CE KENO-VI. These comparisons show excellent agreement between Shift and the accepted benchmark solutions for this problem. This result is expected between these two similar MC CE physics codes. Some representative pin power comparisons using octant symmetry for three Problem 2 lattices are shown in Figs. 19, 20, and 21. These figures show the percent relative difference between Shift pin powers and CE KENO-VI pin powers. Water holes and control rods are shown in black since they have zero power. All of these figures illustrate close agreement between Shift and the benchmark data.

\subsubsection{VERA problem 5}

CASL VERA problem 5 consists of a full reactor model consistent with nuclear core analysis. The model is a full core of Westinghouse $17 \times 17$-type fuel assemblies in the Watts Bar 1 (WBN1) initial loading pattern with all fuel at BOL and hot-zero power (HZP) isothermal conditions. The full problem consists of 32 different reactor configurations simulating various rod bank 
Table 1: Shift eigenvalue and fission rate distribution comparisons for VERA problem 2.

\begin{tabular}{|c|c|c|c|c|c|}
\hline Case & Description & $\begin{array}{l}\text { Ref. } k_{\text {eff }} \\
{[ \pm 3 \mathbf{p c m}]}\end{array}$ & $\begin{array}{c}k_{\text {eff }} \operatorname{diff} \\
{[\mathrm{pcm}]}\end{array}$ & $\begin{array}{c}\text { RMS } \\
{[\%]}\end{array}$ & $\begin{array}{r}\operatorname{Max} \\
{[\%]}\end{array}$ \\
\hline $2 \mathrm{~A}$ & $565 \mathrm{~K}$ & 1.182175 & $42 \pm 6$ & 0.04 & 0.15 \\
\hline $2 \mathrm{~B}$ & $600 \mathrm{~K}$ & 1.183360 & $42 \pm 6$ & 0.03 & 0.06 \\
\hline $2 \mathrm{C}$ & $900 \mathrm{~K}$ & 1.173751 & $36 \pm 5$ & 0.03 & 0.09 \\
\hline $2 \mathrm{D}$ & $1200 \mathrm{~K}$ & 1.165591 & $35 \pm 5$ & 0.02 & 0.05 \\
\hline $2 \mathrm{E}$ & 12 Pyrex & 1.069627 & $29 \pm 5$ & 0.04 & 0.17 \\
\hline $2 \mathrm{~F}$ & 24 Pyrex & 0.976018 & $30 \pm 5$ & 0.04 & 0.09 \\
\hline $2 \mathrm{G}$ & $24 \mathrm{AIC}$ & 0.847695 & $49 \pm 4$ & 0.04 & 0.18 \\
\hline $2 \mathrm{H}$ & $24 \mathrm{~B}_{4} \mathrm{C}$ & 0.788221 & $35 \pm 4$ & 0.04 & 0.14 \\
\hline $2 \mathrm{I}$ & Instrument Thimble & 1.179916 & $38 \pm 6$ & 0.04 & 0.10 \\
\hline $2 \mathrm{~J}$ & Instr. + 24 Pyrex & 0.975193 & $35 \pm 5$ & 0.04 & 0.12 \\
\hline $2 \mathrm{~K}$ & Zoned + 24 Pyrex & 1.020063 & $36 \pm 5$ & 0.03 & 0.08 \\
\hline $2 \mathrm{~L}$ & 80 IFBA & 1.018915 & $27 \pm 5$ & 0.04 & 0.18 \\
\hline $2 \mathrm{M}$ & 128 IFBA & 0.938796 & $43 \pm 5$ & 0.04 & 0.08 \\
\hline $2 \mathrm{~N}$ & 104 IFBA +20 WABA & 0.869615 & $3 \pm 4$ & 0.04 & 0.12 \\
\hline $2 \mathrm{O}$ & $12 \mathrm{Gd}$ & 1.047729 & $35 \pm 5$ & 0.04 & 0.14 \\
\hline $2 \mathrm{P}$ & $24 \mathrm{Gd}$ & 0.927410 & $37 \pm 4$ & 0.05 & 0.14 \\
\hline $2 \mathrm{Q}$ & Zirc4 Spacer Grid & 1.171940 & $32 \pm 5$ & 0.04 & 0.13 \\
\hline \multicolumn{3}{|c|}{ Average } & $34 \pm 5$ & 0.04 & 0.12 \\
\hline
\end{tabular}

positions. Full details of this reactor model and benchmark are given in Refs. [30] and [31].

All core configurations were run using Shift via Insilico for 1000 total cycles with 300 inactive cycles and $1 \times 10^{7}$ particle histories per cycle. These runs were used to compare the eigenvalues computed by Shift with the benchmark CE KENO-VI eigenvalues. The eigenvalue comparison for all cases can be found in Appendix A, Table A.1. The average Shift eigenvalue difference from the benchmark over all cases is $36 \pm 6 \mathrm{pcm}$. Therefore, Shift can accurately predict and calculate the eigenvalue for variations of this reactor core as compared to the benchmark.

The power distribution for this problem was obtained from a separate Shift calculation simulating $1 \times 10^{12}$ total particle histories without in-core instrumentation and with control rod Bank D at the initial critical configura- 


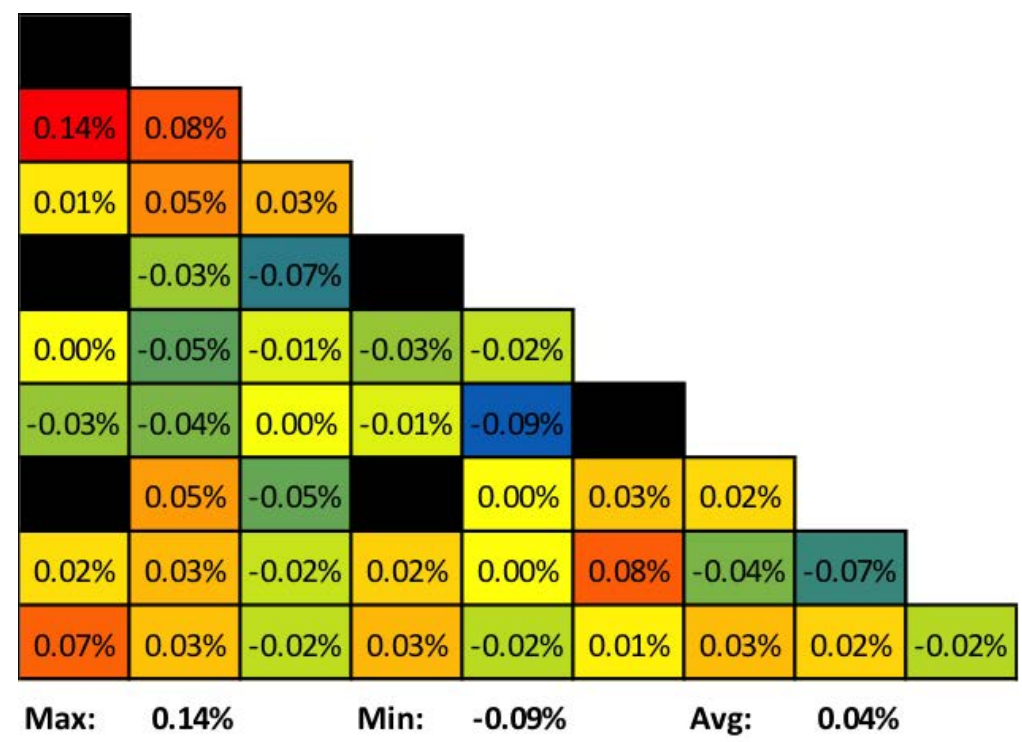

Figure 19: Shift normalized power distribution comparison for VERA problem 2H (AIC) with octant symmetry.

tion. The reference solution contains additional radial core structure, such as the core barrel and neutron pads, that were not included in the Shift model. This exclusion is expected to result in a small deviation in pin and assembly power from Shift in the assemblies closest to the neutron pad on the core diagonal axis.

Figure 22 shows the axially integrated pin power distribution for this configuration. Figure 23 shows the three-dimensional power distribution, and Fig. 24 shows the assembly power distribution. All of these figures give the relative difference between Shift and CE KENO-VI in percent. From these results we conclude that Shift accurately calculates the power distribution in this reactor core as compared to the CE KENO-VI results from the benchmark.

Besides the criticality coefficient and pin powers of these various full reactor model configurations, results obtained from Shift via Insilico were used to calculate rod bank reactivity worths and the differential soluble boron worth. These Shift reactivity and boron worths were compared with the benchmark and with results from various other codes. For each code, including Shift, the measured worths are inferred from the reference bank critical position using calculated shadow factors, and to be consistent, each code was used to calculate these factors. For further details about this comparison, see the 


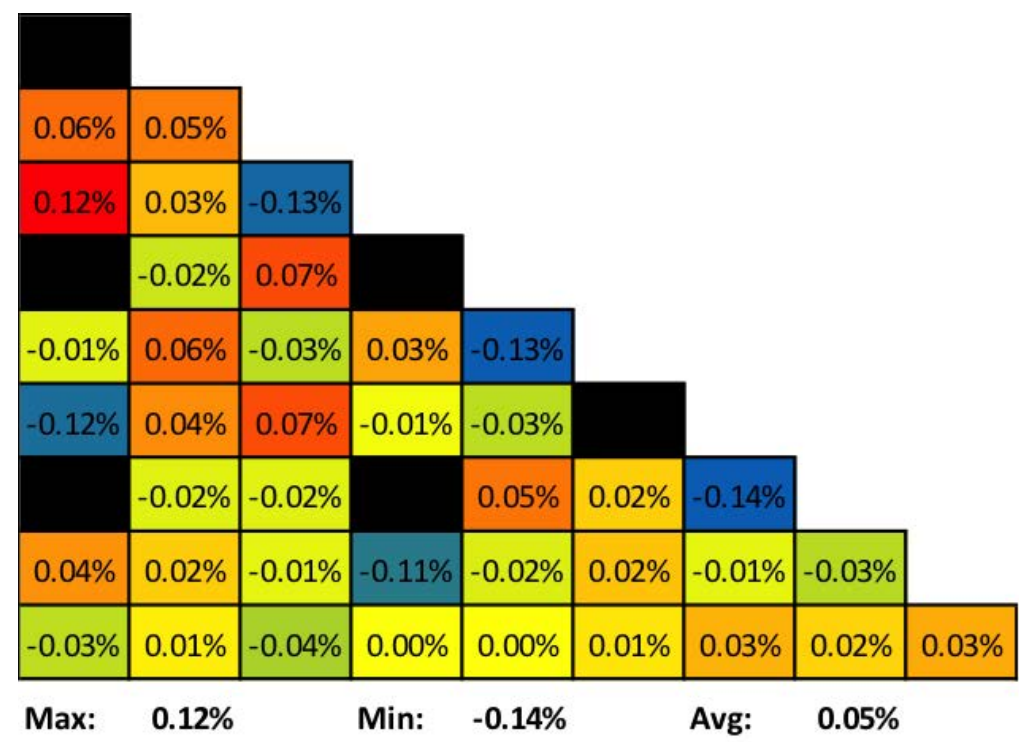

Figure 20: Shift normalized power distribution comparison for VERA problem 2P (Gd) with octant symmetry.

CASL technical report 32 .

Figure 25 shows the comparison of rod bank worth among Shift, the benchmark, and various radiation transport codes. NEXUS is the Westinghouse deterministic radiation transport code, and MPACT is the CASL 2D-1D characteristic deterministic radiation transport code. As expected, Shift, predicts the bank worth with the same relative error as CE KENO-VI. Table 2 shows the differential boron worth calculated using Shift, the benchmark, and other codes. The results in this table clearly show the accuracy of Shift in predicting the differential boron worth for a full reactor core.

Overall, the CASL VERA core physics benchmarks have shown that Shift accurately calculates and predicts quantities of interest for reactor safety and startup.

\subsection{Westinghouse AP1000 ${ }^{\circledR}$ Simulations}

The next set of problems used for Shift reactor physics validation included core physics simulations of the Westinghouse AP1000 ${ }^{\circledR}$ PWR startup core. Details of the AP1000 ${ }^{\circledR}$ PWR and its simulation via the CASL VERA test stand can be found in Refs. [33] and [34]. Reference solutions for this set of benchmarks are taken from CE KENO-VI simulations of the various AP1000 ${ }^{\circledR}$ 


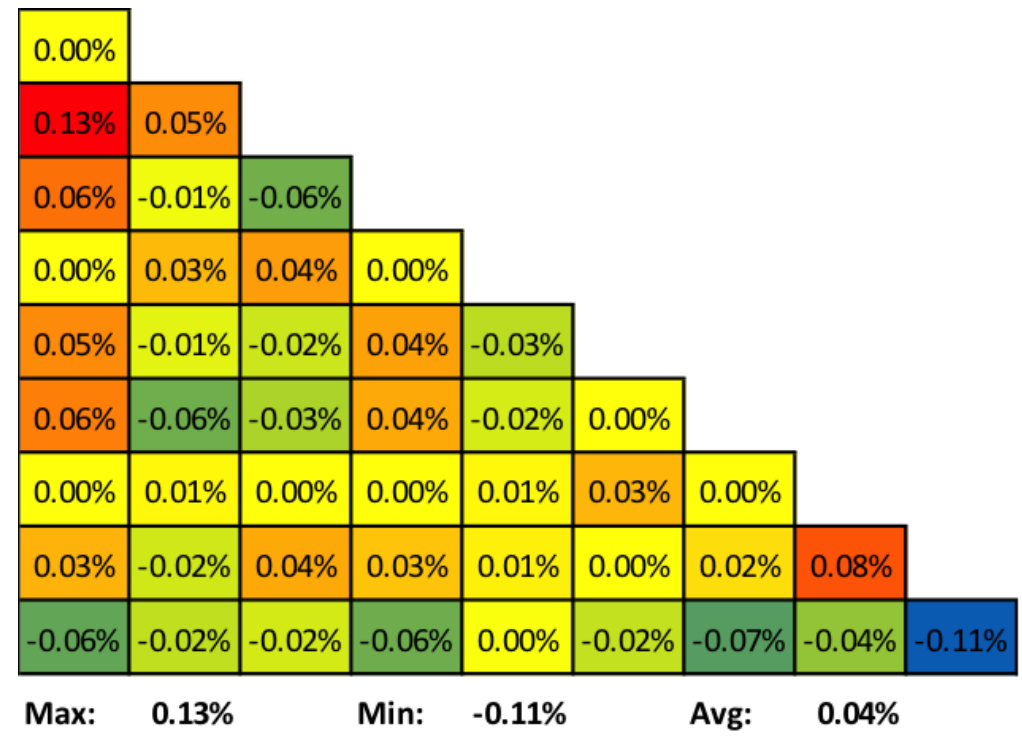

Figure 21: Shift normalized power distribution comparison for VERA problem 2Q (Zr grid) with octant symmetry.

PWR configurations. The Shift simulations of these reactor configurations were performed on Titan to show the advantage of using HPC for reactor safety calculations [23].

Shift-simulated pin powers for this reactor are shown in Fig. 26. This figure shows a cutout of the power distribution in a quarter of the AP $1000^{\circledR}$ core with the $\mathrm{M}$ and $\mathrm{AO}$ control rod banks inserted at different levels. These powers are directly output from Insilico and can be read into VisIt [15] to produce these detailed images and enable finely tuned analysis.

Next, we show an eigenvalue comparison for the various AP1000 ${ }^{\circledR}$ PWR core configurations between Shift and CE KENO-VI. Table 3 shows the eigenvalues from Shift and CE KENO-VI runs along with the difference between the two code results. As expected, Shift eigenvalues agree very well with those from CE KENO-VI, yielding an average difference of $18 \mathrm{pcm}$ and maximum difference of 25 pcm over all of these cases.

Along with the eigenvalue, the power distribution of the AP1000 ${ }^{\circledR}$ ARO (AO control rod bank configuration) problem was calculated using Shift. Table 4 shows the comparison of the assembly and pin power distributions with CE KENO-VI power distributions. From this table, we can conclude that Shift accurately predicts the integrated pin power distributions across 
Table 2: Results and comparisons of differential boron worths for VERA problem 5.

\begin{tabular}{lcc}
\hline Result & $\begin{array}{c}\text { DBW } \\
{[\mathbf{p c m} / \mathbf{p p m B}]}\end{array}$ & $\begin{array}{c}\text { Difference } \\
{[\mathrm{pcm} / \mathbf{p p m B}]}\end{array}$ \\
\hline Measured & -10.77 & -- \\
CE KENO-VI & -10.21 & 0.56 \\
NEXUS & -10.12 & 0.65 \\
Denovo-SPN & -10.16 & 0.61 \\
MPACT & -10.16 & 0.61 \\
Shift & -10.25 & 0.52 \\
\hline
\end{tabular}

this reactor core to less than $1 \%$ error.

This verification problem was also used to look at rod bank worth using Shift. Figure 27 shows the location of the rod banks in a quarter of the AP1000 ${ }^{\circledR}$ core. A comparison of the rod bank worth with CE KENO-VI results is given in Table 5. The largest difference between Shift and CE KENO-VI occurs for a bank closest to the edge of the core with a difference of $2.3 \%$ rod worth with an overall RMS of $1.1 \%$ from all rod bank comparisons.

A strong scaling study of Shift using problem 1 was also performed on Titan. This study used full domain replication, multiple sets with one block per set, of the problem geometry with a fixed number of particle histories per cycle of $5 \times 10^{8}$. Figure 28 shows the excellent efficiency that Shift attains on Titan. An efficiency of $97 \%$ to $100 \%$ is achieved on Titan for this problem. The solve times for the transport solve of these scaling runs are also shown in Fig. 28.

This verification problem set shows that Shift produces detailed solutions that provide insight into understanding start-up core conditions much faster than the current benchmarking tools used by CASL. We have shown that Shift can achieve excellent scaling on a leadership-class HPC machine such as Titan.

\subsection{NEA MC Performance Benchmark Results}

To show how Shift performs for a full reactor calculation, we ran the Nuclear Energy Agency (NEA) MC performance benchmark for detailed power density calculation in a full size reactor core [28]. The details of the benchmark specification can be found in reference [28]. Shift was run using an MCNP5 geometry and simulated $1 \times 10^{9}$ particle histories per cycle for a 
Table 3: Shift eigenvalue and benchmark comparison results for different assemblies in AP1000 ${ }^{\circledR}$.

\begin{tabular}{cccc}
\hline Case ID & KENO-VI $k_{\text {eff }}$ & Shift $k_{\text {eff }}$ & Diff $[\mathbf{p c m}]$ \\
\hline ARO & 1.000870 & 1.001030 & 16 \\
DBW & 1.003240 & 1.003450 & 21 \\
MA & 0.998258 & 0.998414 & 16 \\
MB & 0.998669 & 0.998909 & 24 \\
MC & 0.998956 & 0.999148 & 19 \\
MD & 0.998496 & 0.998643 & 15 \\
M1 & 0.994350 & 0.994548 & 20 \\
M2 & 0.992001 & 0.992185 & 18 \\
AO & 0.984609 & 0.984749 & 14 \\
S1 & 0.990103 & 0.990200 & 10 \\
S2 & 0.989935 & 0.990183 & 25 \\
S3 & 0.989650 & 0.989739 & 9 \\
S4 & 0.995055 & 0.995295 & 24 \\
\hline Average & & & $\mathbf{1 8}$ \\
\hline
\end{tabular}

total of $1 \times 10^{3}$ cycles. The number of inactive cycles run was 300 to begin tallying after fission source convergence.

Shift was run with a mesh tallying power covering the entire reactor core with approximately $1.13 \times 10^{7}$ mesh cells, including the $6.3624 \times 10^{6}$ power densities requested by the benchmark. This problem was run on Titan using $1 \times 10^{5}$ processors split across 12,500 nodes ( 8 processors per node) and used a walltime of 3.43 hours. Shift produced a power density solution with less than $1 \%$ relative error in $98 \%$ of all mesh cells tallied, with all power densities calculated with less than $3 \%$ relative error.

A strong scaling study of this benchmark was also performed using Shift on Titan. This study used full domain replication of the problem geometry with a fixed humber of particle histories per cycle of $1 \times 10^{7}$. Figure 29 shows the excellent efficiency Shift attains on Titan for this benchmark. An efficiency of $91 \%$ to $100 \%$ is achieved. This figure also shows the solve times for the transport solve of these runs.

In comparison with the scaling achieved for the AP1000 ${ }^{\circledR}$ problem on Titan, note that the number of particle histories per cycle on each processor 
Table 4: Comparisons of eigenvalues and overall pin powers, $\Delta \mathrm{P}$, for AP1000 ${ }^{\circledR}$.

\begin{tabular}{|c|c|c|c|c|c|c|c|}
\hline Code & $\begin{array}{c}k_{\text {eff }} \\
\pm 2 \mathrm{pcm} \\
\end{array}$ & $\begin{array}{r}\Delta k_{\mathrm{eff}} \\
{[\mathrm{pcm}]}\end{array}$ & $\begin{array}{c}\text { RMS } \\
\text { Asm. } \\
{[\%]}\end{array}$ & $\begin{array}{c}\text { Max } \\
\text { Asm. } \\
{[\%]}\end{array}$ & $\begin{array}{c}\text { RMS } \\
\text { Pin } \\
{[\%]}\end{array}$ & $\begin{array}{c}\text { Max } \\
\text { Pin } \\
{[\%]}\end{array}$ & $\begin{array}{l}\text { Hot } \\
\text { Pin } \\
{[\%]} \\
\end{array}$ \\
\hline KENO-VI & 1.00096 & - & - & - & - & - & 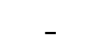 \\
\hline Denovo & 1.00086 & -10 & 0.2 & 0.4 & 0.4 & 3.0 & 1.0 \\
\hline Shift & 1.00131 & 35 & 0.2 & 0.5 & 0.2 & 0.6 & -0.2 \\
\hline
\end{tabular}

for this problem reaches a minimum of approximately 300 versus a minimum of approximately 3300 for the $\mathrm{AP} 1000^{\circledR}$ problem. This small number of particle histories per core leads to a slight degradation in parallel efficiency because communication costs become more dominant.

Through this performance benchmark, we have shown that Shift achieves excellent scaling on a leadership-class machine for a typical full reactor simulation. We have also shown that Shift can attain a solution for a full reactor simulation with minimal statistical uncertainty in a quick timeframe.

In summary, we have shown some of the validation and verification performed with Shift, its performance, and how it compares to other advanced radiation transport codes.

\section{Conclusions}

Shift was designed to efficiently tackle a wide class of applications. To suit this purpose, the transport components in Shift have been designed to be agnostic to both geometry and physics. This allows the incorporation of multiple geometry and physics implementations with minimal integration effort. Supporting multiple geometry and physics specifications allows Shift to execute problems in many application areas.

Shift was designed to run on a wide range of computational hardware, ranging from laptops to leadership-class machines. Full core analysis, including depletion, will present significant memory stresses on even the largest machines; therefore, we have developed the MSOD parallel algorithm to allow scaling and smooth transitions between fully domain-replicated topologies and spatially domain-decomposed problems. Using the MSOD algorithm, we have demonstrated excellent strong scaling results for multiset, singleblock configurations. We have also shown good weak scaling using multiset, 
Table 5: Comparison of rod bank worth calculated using Shift to the benchmark for $\operatorname{AP} 1000^{\circledR}$.

\begin{tabular}{cccrr}
\hline \multirow{2}{*}{ Rod Bank } & \multirow{2}{*}{ Material } & KENO-VI & \multicolumn{2}{c}{ Shift $\Delta$ Worth } \\
& & Worth $[\mathbf{p c m}]$ & {$[\mathbf{p c m}]$} & {$[\%]$} \\
\hline MA & Tungsten & 258 & 4 & 1.4 \\
MB & Tungsten & 217 & -5 & -2.3 \\
MC & Tungsten & 188 & 0 & 0.1 \\
MD & Tungsten & 234 & 5 & 2.0 \\
M1 & Ag-In-Cd & 651 & -1 & -0.2 \\
M2 & Ag-In-Cd & 887 & 1 & 0.1 \\
AO & Ag-In-Cd & 1635 & 5 & 0.3 \\
S1 & Ag-In-Cd & 1079 & 9 & 0.8 \\
S2 & Ag-In-Cd & 1096 & -7 & -0.6 \\
S3 & Ag-In-Cd & 1124 & 10 & 0.9 \\
S4 & Ag-In-Cd & 580 & 6 & 0.1 \\
\hline & & RMS & $\mathbf{6}$ & $\mathbf{1 . 1}$ \\
& & Max & $\mathbf{1 0}$ & $\mathbf{2 . 3}$ \\
\hline
\end{tabular}

multiblock decompositions.

The development of Shift over the last year has focused on solving problems of interest to CASL, namely LWR core physics. Thus, in order for Shift to be useful in this context, we have performed extensive benchmarking on LWR-relevant problems. The benchmarking and other results discussed in this paper have successfully validated and verified Shift for LWR core-analysis applications.

\section{Acknowledgments}

Thanks to Fausto Franceschini at Westinghouse for contributing some of the AP $1000{ }^{\circledR}$ figures and data.

Work for this paper was supported by Oak Ridge National Laboratory, which is managed and operated by UT-Battelle, LLC, for the U.S. Department of Energy under Contract No. DEAC05-00OR22725. This research was supported by the Consortium for Advanced Simulation of Light Water Reactors (www.casl.gov), an Energy Innovation Hub (http://www.energy.gov/hubs) for Modeling and Simulation of Nuclear Reactors under U. S. Department 
of Energy Contract No. DE-AC05-00OR22725. This research was also sponsored by the Laboratory Directed Research and Development Program of Oak Ridge National Laboratory, managed by UT-Battelle, LLC, for the U. S. Department of Energy. This research used resources of the Oak Ridge Leadership Computing Facility at the Oak Ridge National Laboratory, which is supported by the Office of Science of the U.S. Department of Energy under Contract No. DE-AC05-00OR22725.

\section{Appendix A. CASL VERA Problem 5 Eigenvalue Comparison}

This section of the appendix gives the raw eigenvalue comparison of the Shift results for each VERA Problem 5 case. These results and comparisons are given in Table A.1. All of the Shift $k_{\text {eff }}$ differences given in this table have an error of $\pm 6 \mathrm{pcm}$. 
Table A.1: Shift eigenvalue results and comparison for VERA problem 5.

\begin{tabular}{|c|c|c|c|c|c|c|c|c|c|c|c|}
\hline & \multirow{2}{*}{$\begin{array}{c}\mathrm{B} \\
{[\mathrm{ppm}]}\end{array}$} & \multicolumn{8}{|c|}{ Bank position (steps withdrawn) } & \multirow{2}{*}{$\begin{array}{c}\text { Ref } \\
<2[\mathrm{pcm}]\end{array}$} & \multirow{2}{*}{$\begin{array}{r}\Delta k_{\text {eff }} \\
{[\mathrm{pcm}}\end{array}$} \\
\hline & & $\mathbf{A}$ & B & $\mathrm{C}$ & $\mathrm{D}$ & $\mathrm{SA}$ & SB & $\mathrm{SC}$ & SD & & \\
\hline 1 & 1285 & - & - & - & 167 & - & - & - & - & 0.9999 & 34 \\
\hline 2 & 1291 & - & - & - & - & - & - & - & - & 1.0003 & 36 \\
\hline 3 & 1170 & 0 & - & - & 97 & - & - & - & - & 0.9988 & 26 \\
\hline 4 & $\downarrow$ & - & 0 & - & 113 & - & - & - & - & 0.9994 & 37 \\
\hline 5 & $\downarrow$ & - & - & 0 & 119 & - & - & - & - & 0.9990 & 27 \\
\hline 6 & $\downarrow$ & - & - & - & 18 & - & - & - & - & 0.9991 & 37 \\
\hline 7 & $\downarrow$ & - & - & - & 69 & 0 & - & - & - & 0.9990 & 33 \\
\hline 8 & $\downarrow$ & - & - & - & 134 & - & 0 & - & - & 0.9993 & 37 \\
\hline 9 & $\downarrow$ & - & - & - & 71 & - & - & 0 & - & 0.9989 & 30 \\
\hline 10 & $\downarrow$ & - & - & - & 71 & - & - & - & 0 & 0.9990 & 32 \\
\hline 11 & $\downarrow$ & - & - & - & - & - & - & - & - & 1.0128 & 40 \\
\hline 12 & $\downarrow$ & 0 & - & - & - & - & - & - & - & 1.0037 & 35 \\
\hline 13 & $\downarrow$ & - & 0 & - & - & - & - & - & - & 1.0039 & 41 \\
\hline 14 & $\downarrow$ & - & - & 0 & - & - & - & - & - & 1.0028 & 34 \\
\hline 15 & $\downarrow$ & - & - & - & 0 & - & - & - & - & 0.9988 & 39 \\
\hline 16 & $\downarrow$ & - & - & - & - & 0 & - & - & - & 1.0083 & 45 \\
\hline 17 & $\downarrow$ & - & - & - & - & - & 0 & - & - & 1.0020 & 38 \\
\hline 18 & $\downarrow$ & - & - & - & - & - & - & 0 & - & 1.0077 & 39 \\
\hline 19 & $\downarrow$ & - & - & - & - & - & - & - & 0 & 1.0077 & 41 \\
\hline 22 & 1230 & - & - & - & 0 & - & - & - & - & 0.9928 & 32 \\
\hline 23 & $\downarrow$ & - & - & - & 23 & - & - & - & - & 0.9932 & 32 \\
\hline 24 & $\downarrow$ & - & - & - & 46 & - & - & - & - & 0.9946 & 34 \\
\hline 25 & $\downarrow$ & - & - & - & 69 & - & - & - & - & 0.9974 & 32 \\
\hline 26 & $\downarrow$ & - & - & - & 92 & - & - & - & - & 1.0003 & 36 \\
\hline 27 & $\downarrow$ & - & - & - & 115 & - & - & - & - & 1.0025 & 34 \\
\hline 28 & $\downarrow$ & - & - & - & 138 & - & - & - & - & 1.0042 & 38 \\
\hline 29 & $\downarrow$ & - & - & - & 161 & - & - & - & - & 1.0053 & 37 \\
\hline 30 & $\downarrow$ & - & - & - & 184 & - & - & - & - & 1.0061 & 37 \\
\hline 31 & $\downarrow$ & - & - & - & 207 & - & - & - & - & 1.0065 & 39 \\
\hline 32 & $\downarrow$ & - & - & - & - & - & - & - & - & 1.0066 & 37 \\
\hline \multicolumn{2}{|c|}{ Average } & & & & & & & & & & 36 \\
\hline
\end{tabular}




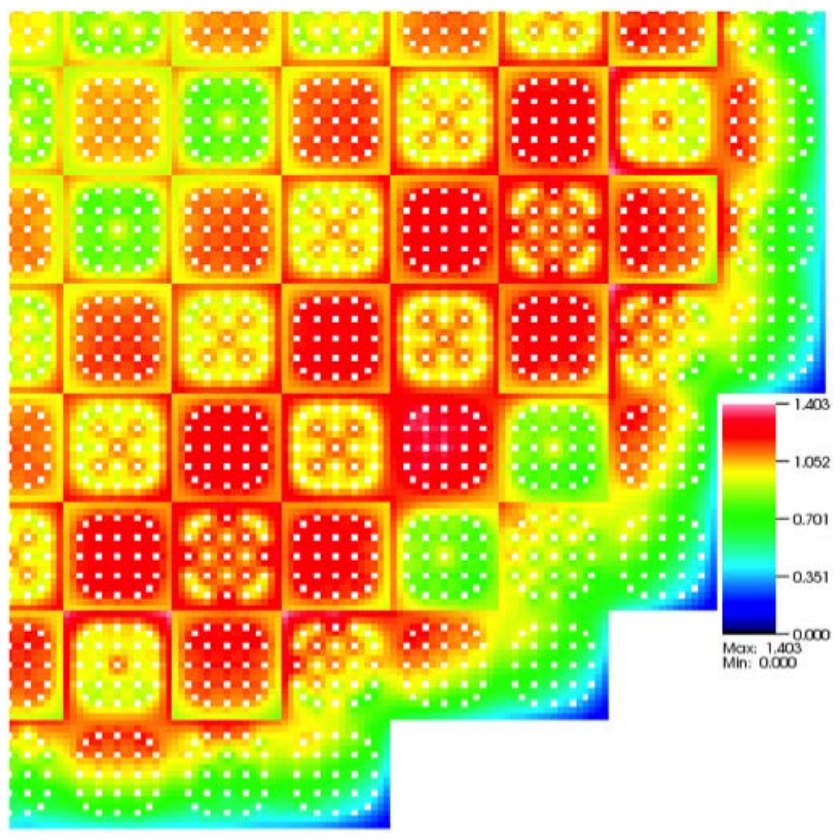

(a) Powers.

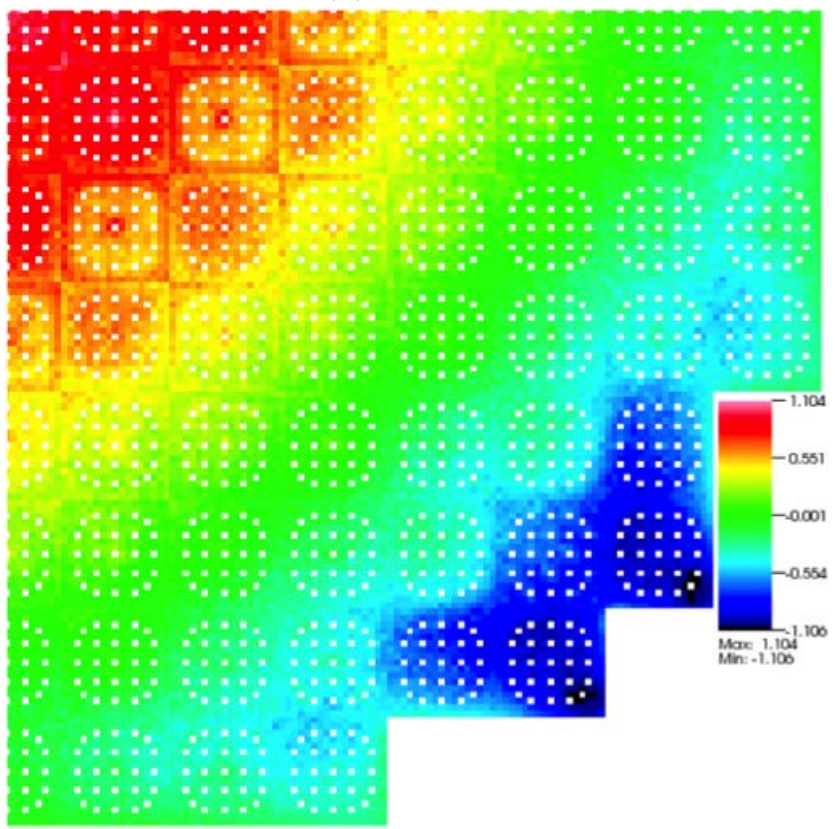

(b) Relative differences with CE KENO-VI[\%]; RMS $=0.46 \%$.

Figure 22: Axially-integrated normalized pin power distributions from Shift for VERA problem 5. 


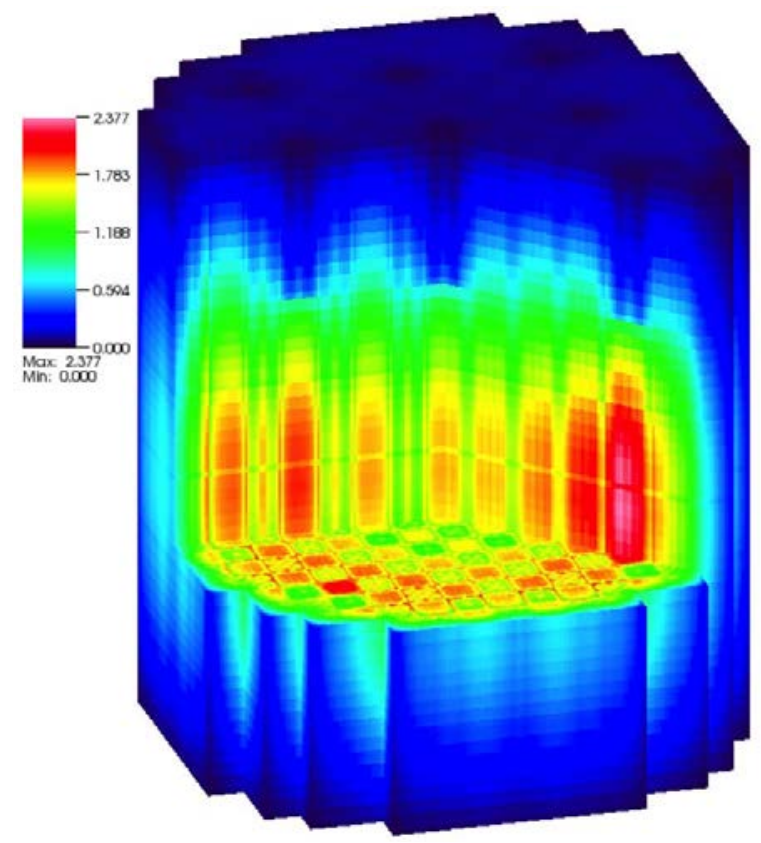

(a) Powers.

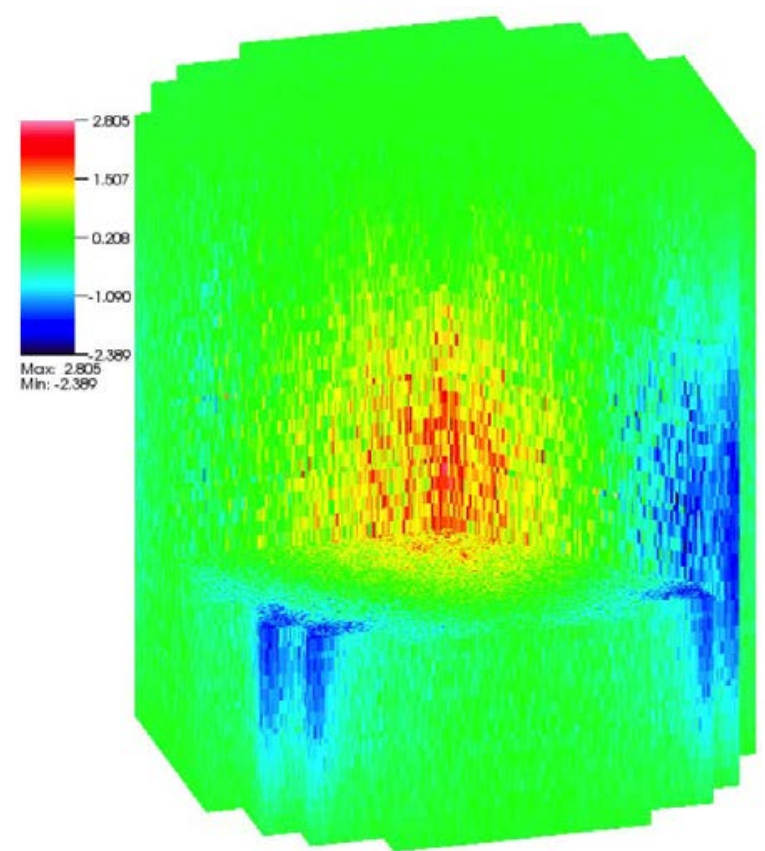

(b) Relative differences with CE KENO-VI[\%]; RMS $=0.56 \%$.

Figure 23: Full-core normalized pin power distributions from Shift for VERA problem 5. 


\begin{tabular}{|c|c|c|c|c|c|}
\hline 0.958 & & & & & \\
\hline 0.928 & 1.006 & & & & \\
\hline 1.026 & 0.915 & 1.071 & & & \\
\hline 0.991 & 1.087 & 1.045 & 1.164 & & \\
\hline 1.069 & 1.050 & 1.176 & 1.085 & 1.234 & \\
\hline 1.050 & 1.163 & 1.152 & 1.148 & 0.893 & 0.905 \\
\hline 1.084 & 1.065 & 1.101 & 1.045 & 0.939 & 0.621 \\
\hline 0.792 & 0.905 & 0.801 & 0.655 & & \\
\hline
\end{tabular}

Max: 1.234

Avg: 1.000

(a) Powers.

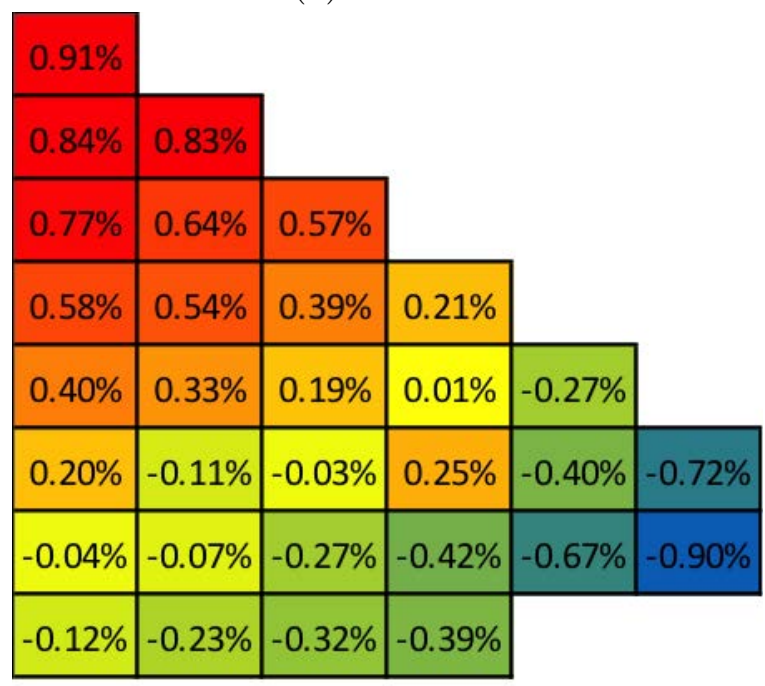

Max: $0.84 \% \quad$ Min: $-0.90 \% \quad$ RMS: $0.45 \%$

(b) Relative differences with CE KENO-VI.

Figure 24: Assembly normalized pin power distributions from Shift for VERA problem 5. 
Figure 25: Various rod bank worths for VERA problem 5.

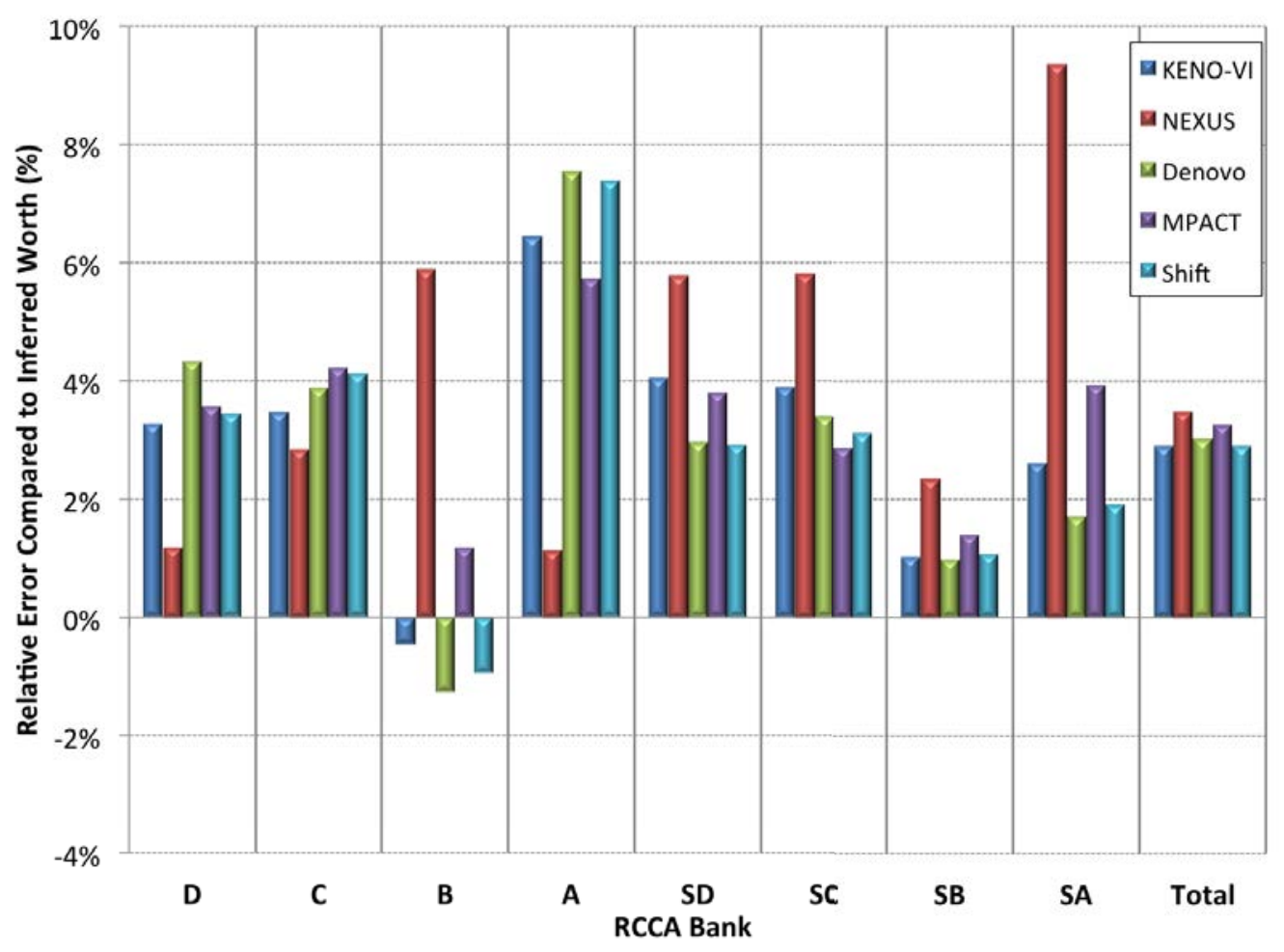




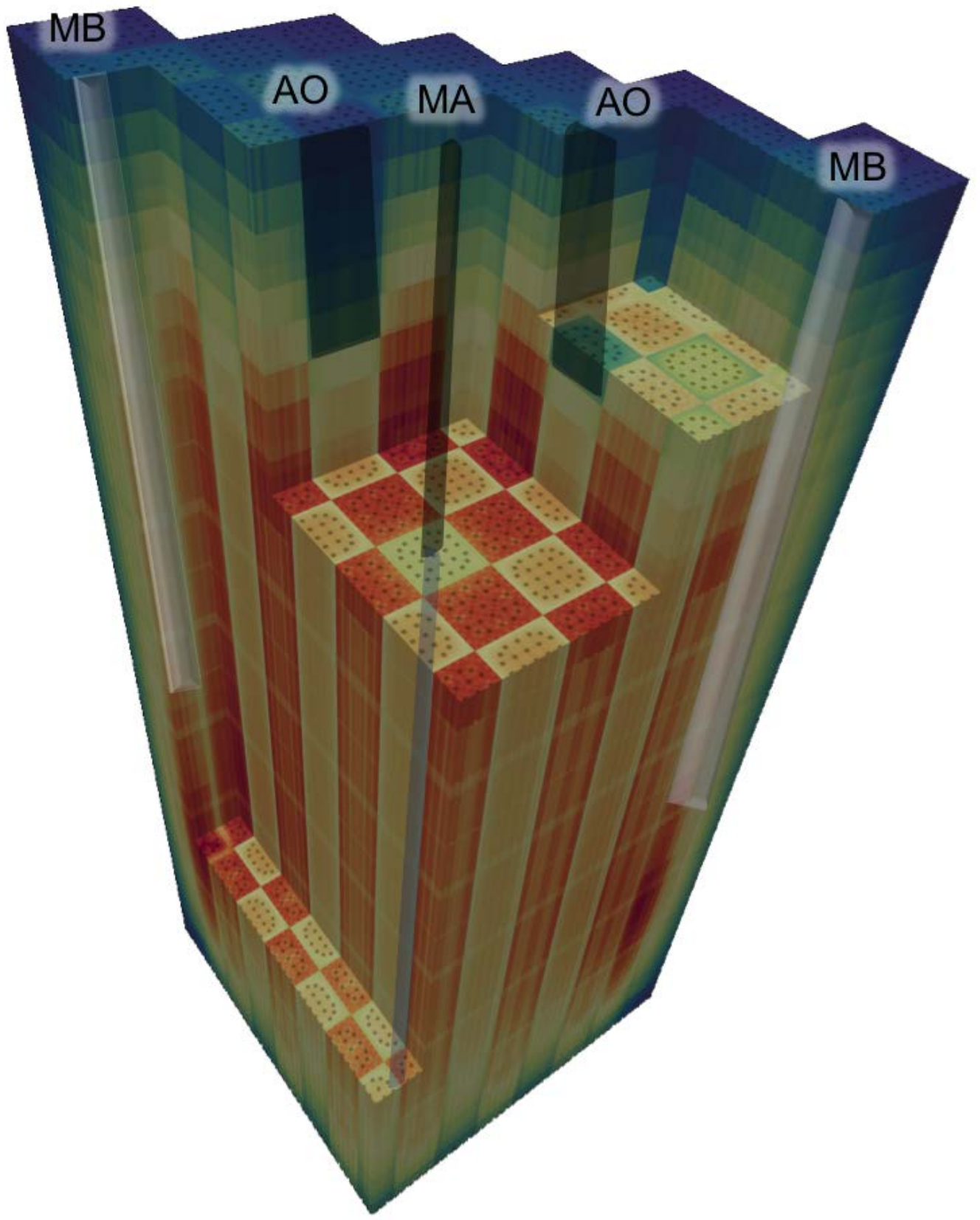

Figure 26: Shift power distribution (with AO and M-banks inserted) for AP1000 ${ }^{\circledR}$. 


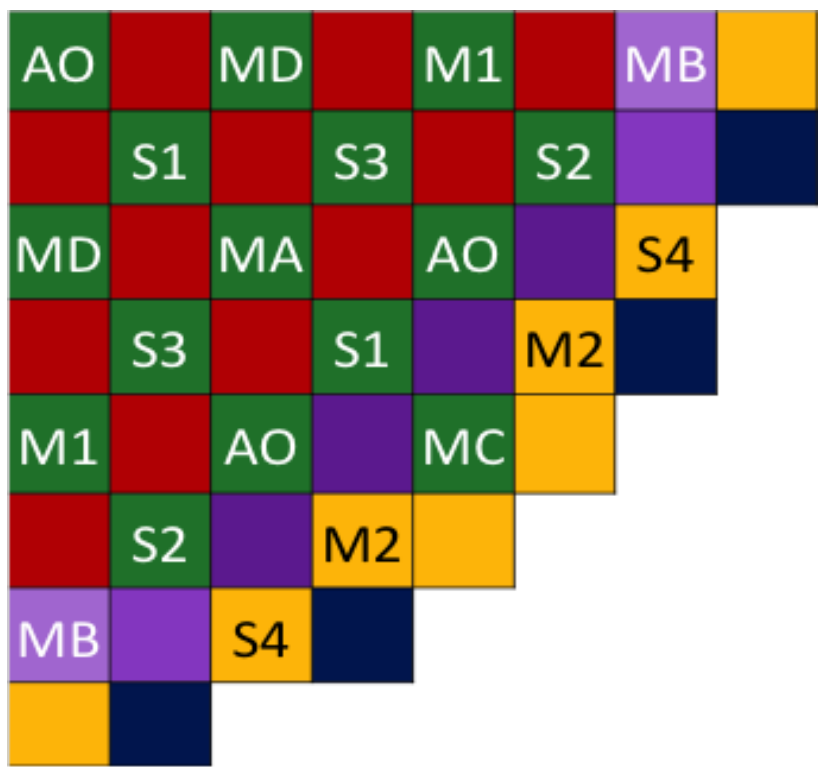

Figure 27: Rod bank locations in AP1000 ${ }^{\circledR}$ (image produced from Shift run). 


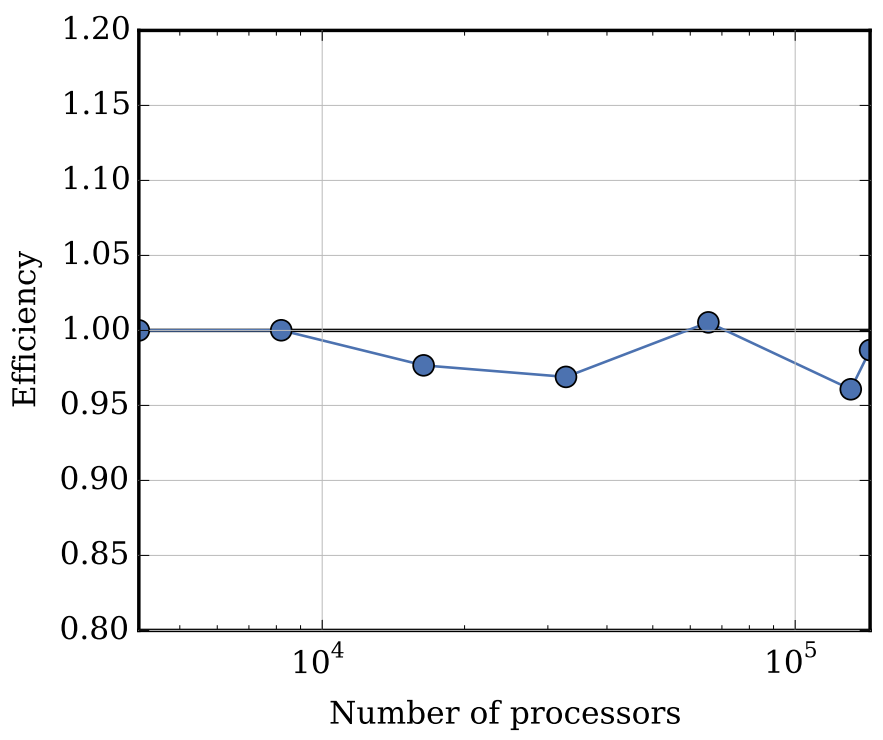

(a) Efficiency

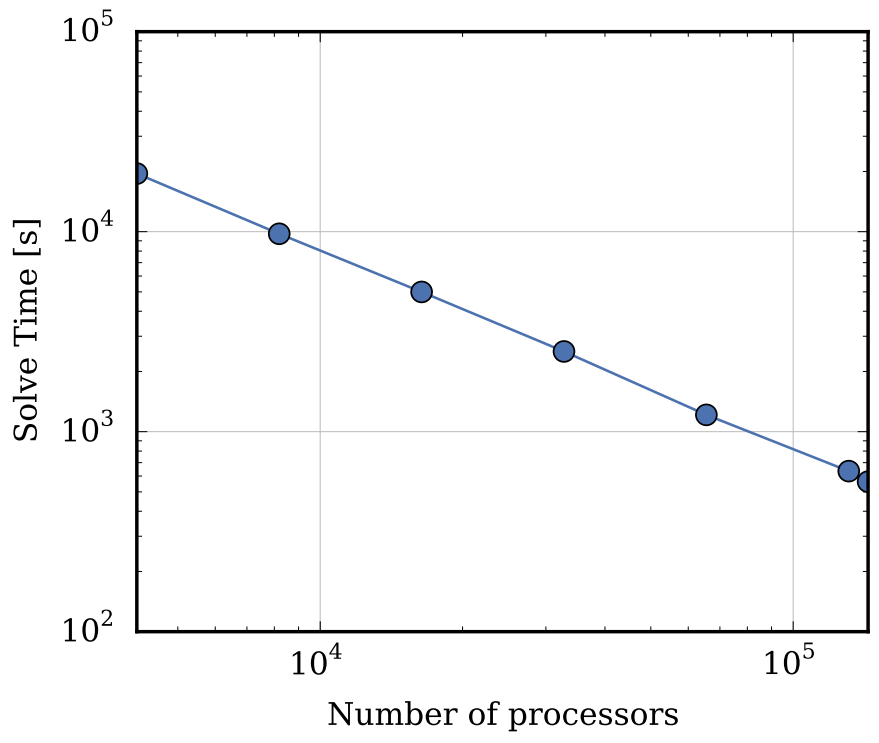

(b) Solve Time

Figure 28: Shift strong scaling results for AP1000 ${ }^{\circledR}$ problem 1 run on Titan. 


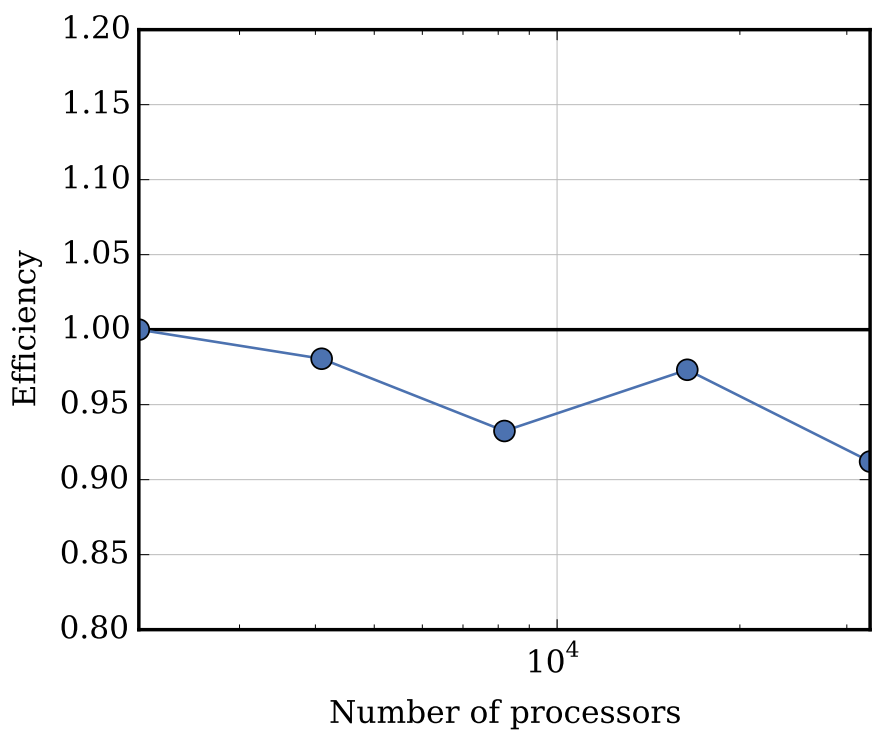

(a) Efficiency

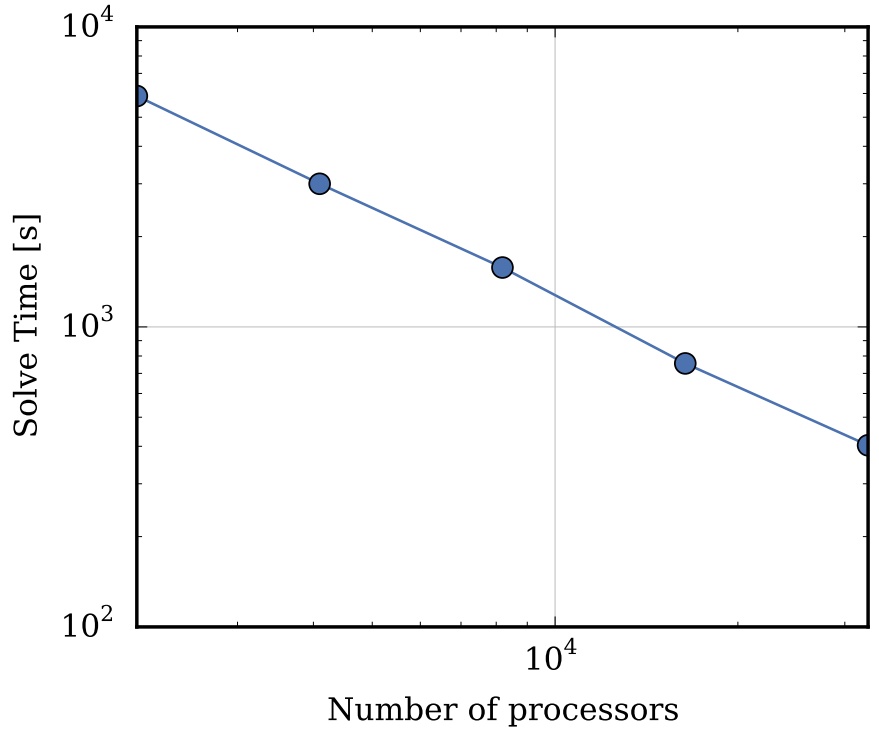

(b) Solve Time

Figure 29: Shift strong scaling results for the NEA MC performance benchmark for reactor calculations run on Titan. 


\section{References}

[1] T. M. Evans, A. S. Stafford, R. Slaybaugh, K. T. Clarno, Denovo: A new three-dimensional parallel discrete ordinates code in SCALE, Nuclear Technology 171 (2010) 171-200.

[2] J. J. Jarrell, T. M. Evans, G. G. Davidson, A. T. Godfrey, Full Core Reactor Analysis : Running Denovo on Jaguar, Nuclear Science and Engineering 175 (2013) 283-291.

[3] CASL: Improving reactor performance with predictive science-based simulation technology that harnesses world-class computational power, Tech. Rep. CASL-U-2014-0089-000, Oak Ridge National Laboratory (2014).

[4] R. D. Cheverton, T. M. Sims, HFIR Core Nuclear Design, Tech. Rep. ORNL-4621, Oak Ridge National Laboratory (1971).

[5] E. D. Cashwell, C. J. Everett, A Practical Manual on the Monte Carlo Method for Random Walk Problems, Tech. Rep. LA-2120, Los Alamos Scientific Laboratory (1957).

[6] L. L. Carter, E. D. Cashwell, Particle-transport simulation with the Monte Carlo method, Tech. Rep. 26607, Los Alamos Scientific Laboratory (1975).

[7] SCALE: A Comprehensive Modeling and Simulation Suite for Nuclear Safety Analysis and Design, Tech. Rep. ORNL/TM-2005/39, Version 6.1, Oak Ridge National Laboratory (2011).

[8] X-5 Monte Carlo Team, MCNP - a general Monte Carlo n-particle transport code, version 5, Tech. Rep. LA-UR-03-1987, Los Alamos National Laboratory (2008).

[9] T. J. Urbatsch, Iterative acceleration methods for Monte Carlo and deterministic criticality calculations, Thesis LA-13052-T, Los Alamos National Laboratory (November 1995).

[10] R. Montgomery, VERA tools and workflows, Technical Report CASL-U2014-0054-001, Oak Ridge National Laboratory (March 2014). 
[11] S. W. Mosher, A. M. Bevill, S. R. Johnson, A. M. Ibrahim, C. R. Daily, T. M. Evans, J. C. Wagner, J. O. Johnson, R. E. Grove, ADVANTGAn Automated Variance Reduction Parameter Generator, Tech. Rep. ORNL/TM-2013/416, Oak Ridge National Laboratory (2013).

[12] M. B. Emmett, MORSE-CGA: A General Purpose Monte Carlo Multigroup Neutron and Gamma-Ray Transport Code System with Array Geometry Capability, Version 2, Tech. Rep. ORNL-6174, Oak Ridge National Laboratory (1985).

[13] Cross Sections Evaluation Working Group, ENDF-6 Formats Manual: Data Formats and Procedures for the Evaluated Nuclear Data Files ENDF/B-VI and ENDF/B-VII, Brookhaven National Laboratory, 2nd Edition (December 2011).

[14] M. E. Dunn, N. M. Greene, AMPX-2000: A Cross-Section Processing System for Generating Nuclear Data for Criticality Safety Applications, Trans. Am. Nucl. Soc. 86 (2002) 118.

[15] H. Childs, E. Brugger, B. Whitlock, J. Meredith, S. Ahern, D. Pugmire, K. Biagas, M. Miller, C. Harrison, G. H. Weber, H. Krishnan, T. Fogal, A. Sanderson, C. Garth, E. W. Bethel, D. Camp, O. Rübel, M. Durant, J. M. Favre, P. Navrátil, VisIt: An End-User Tool For Visualizing and Analyzing Very Large Data, High Performance Visualization-Enabling Extreme-Scale Scientific Insight (2012) 357-372.

[16] T. Ueki, F. B. Brown, Stationarity modeling and informatics-based diagnostics in Monte Carlo criticality calculations, Nucl. Sci. Eng. 149 (1) (2005) 38-50.

[17] J. Wagner, A. Haghighat, Automated variance reduction of monte carlo shielding calculations using the discrete ordinates adjoint function, $\mathrm{Nu}-$ clear Science and Engineering 128 (2) (1998) 186-208.

[18] S. R. Johnson, Fast mix table construction for material discretization, Proc. Intl. Conf. on Math. and Comput. Methods Applied to Nucl. Sci. Eng. (M\&C 2013), Sun Valley, ID, May 5-9.

[19] J. C. Wagner, S. W. Mosher, T. M. Evans, D. E. Peplow, J. A. Turner, Hybrid and parallel domain-decomposition methods development to 
enable Monte Carlo for reactor analyses, Progress in Nuclear Science Technology 2 (2011) 815-820.

[20] T. A. Brunner, T. J. Urbatsch, T. M. Evans, N. A. Gentile, Comparison of four parallel algorithms for domain decomposed implicit Monte Carlo, Journal of Computational Physics 212 (2006) 527-539.

[21] T. A. Brunner, P. S. Brantley, An efficient, robust, domain-decomposition algorithm for particle Monte Carlo, Journal of Computational Physics 228 (2009) 3882-3890.

[22] P. Romano, B. Forget, Parallel fission bank algorithms in Monte Carlo criticality calculations, Nuclear Science and Engineering 170 (2012) 125135 .

[23] Oak Ridge Leadership Computing Facility, Titan Cray XK7 (January 2015). URL https://www.olcf.ornl.gov/computing-resources/ titan-cray-xk7/

[24] VESTA User's Manual - Version 2.1.0, Tech. Rep. DSU/SEC/T/2008-331, IRSN, BP 17, 92262 Fontenay-aux-roses, Cedex, France (2008).

[25] H. R. Trellue, D. I. Poston, User's Manual, Version 2.0 for Monteburns, Version 1.0, Tech. Rep. LA-UR-99-4999, LANL (September 1999).

[26] A. E. Isotalo, V. Sahlberg, Comparison of neutronics-depletion schemes for burnup calculations, Nuclear Science and Engineering 179 (4) (2015) 434-459.

[27] A. E. Isotalo, Comparison of neutronics-depletion coupling schemes for burnup calculations 2, Nuclear Science and Engineering 180 (3) (2015) 286-300.

[28] J. Hoogenboom, W. Martin, B. Petrovic, Monte Carlo Performance Benchmark for Detailed Power Density Calculation in a Full Size Reactor Core, Benchmark Specifications Revision 1.2, http://www.nea.fr/dbprog/MonteCarloPerformanceBenchmark.htm (2011). 
[29] L. W. Newman, W. A. Wittkopf, W. G. Pettus, M. N. Baldwin, H. A. Hassan, V. O. Uotinen, J. D. Connell, P. S. Campbell, Urania-Gadolinia: Nuclear model development and critical experiment benchmark, Tech. Rep. DOE/ET/34212-41 BAW-1810, Babcock and Wilcox Co., Lynchburg, VA (April 1984).

[30] A. T. Godfrey, VERA Core Physics Benchmark Progression Problem Specifications, Tech. Rep. CASL-U-2012-0131-003, CASL (March 2014).

[31] J. C. Gehin, A. T. Godfrey, F. Franceschini, T. M. Evans, B. Collins, S. Hamilton, Operational Reactor Model Demonstration with VERA: Watts Bar Unit 1 Cycle 1 Zero Power Physics Tests, Tech. Rep. CASLU-2013-0105-001, CASL (August 2013).

[32] A. T. Godfrey, MPACT Testing and Benchmarking Results, Tech. Rep. CASL-U-2014-0045-000, CASL (March 2014).

[33] F. Franceschini, A. T. Godfrey, J. Kulesza, R. Oelrich, Westinghouse VERA Test Stand: Zero Power Physics Test Simulations for the AP1000 PWR, Tech. Rep. CASL-U-2014-0012-000, CASL (March 2014).

[34] F. Franceschini, B. Oelrich, J. C. Gehin, Simulation of AP1000 first core with VERA, NEI Magazine. 